\title{
Atmosphere and Ocean Modeling on Grids of Variable Resolution-A 2D Case Study
}

\author{
PETER D. DÜBEN \\ Max Planck Institute for Meteorology and IMPRS-ESM, Hamburg, Germany \\ PETER KORN \\ Max Planck Institute for Meteorology, Hamburg, Germany
}

(Manuscript received 8 July 2013, in final form 17 December 2013)

\begin{abstract}
Grids of variable resolution are of great interest in atmosphere and ocean modeling as they offer a route to higher local resolution and improved solutions. On the other hand there are changes in grid resolution considered to be problematic because of the errors they create between coarse and fine parts of a grid due to reflection and scattering of waves. On complex multidimensional domains these errors resist theoretical investigation and demand numerical experiments. With a low-order hybrid continuous/discontinuous finiteelement model of the inviscid and viscous shallow-water equations a numerical study is carried out that investigates the influence of grid refinement on critical features such as wave propagation, turbulent cascades, and the representation of geostrophic balance. The refinement technique the authors use is static $h$ refinement, where additional grid cells are inserted in regions of interest known a priori. The numerical tests include planar and spherical geometry as well as flows with boundaries and are chosen to address the impact of abrupt changes in resolution or the influence of the shape of the transition zone. For the specific finite-element model under investigation, the simulations suggest that grid refinement does not deteriorate geostrophic balance and turbulent cascades and the shape of mesh transition zones appears to be less important than expected. However, the results show that the static local refinement is able to reduce the local error, but not necessarily the global error and convergence properties with resolution are changed. The relatively simple tests already illustrate that grid refinement has to go along with a simultaneous change of the parameterization schemes.
\end{abstract}

\section{Introduction}

In the numerical modeling of the atmospheric or oceanic general circulation, the affordable resolution is still far from satisfying. This motivates the investigation of local grid refinement as a tool to gain efficiency. Static, local grid refinement could be used to increase the resolution in a specific region of interest, such as a single country or continent, arctic or tropical regions, or to improve the representation of important local features that have a large impact on the whole simulation, such as boundary currents, sea overflows, islands, and deep water formations in an ocean model, and mountains, tropical cyclones, and convection areas in an atmosphere model. A complete treatment of local grid

Corresponding author address: Peter D. Düben, AOPP, Clarendon Laboratory, University of Oxford, Parks Road, Oxford, OX1 3PU, United Kingdom.

E-mail: dueben@atm.ox.ac.uk refinement for atmosphere/ocean models has to address manifold challenges. The first challenges are within the dynamical core. The fluid dynamical part that solves the equations of motion has to be enabled to operate efficiently on refined grids in the horizontal as well as in the vertical direction, where one has to take into account that the refinement in horizontal and eventually in vertical direction will influence each other. The second challenge is the parameterization schemes of subgridscale processes and turbulent closure, which have to take into account the variable resolution. A third challenge is related to the evaluation of the locally refined model. Ideally one would like to have a model that is much "cheaper" than the global model in high resolution but delivers a solution that is, at least in the refined region, "comparable" to the high-resolution solution. The metrics to quantify this in terms of efficiency and in terms of solution quality are far from obvious.

The purpose of this paper is to study the influence of static grid refinement on fundamental features of 
geophysical modeling, such as wave propagation, the representation of turbulent cascades, boundary currents, or geostrophic adjustment. These problems can be addressed in a two-dimensional setup. With respect to the aforementioned challenges we focus on the horizontal component of the dynamical core and consider (two dimensional) horizontally refined grids. These grids are created a priori, they are fixed and not adapted during the experiments. Since the horizontal component of an atmosphere/ocean model resembles to some extent the shallow-water equations, we use these equations as model equations. The questions of horizontal in combination with vertical refinement and the problem of subgrid-scale closures constitute scientific problems in its own and are not addressed here. We also omit the important and intricate topic of comparing the computational costs between refined and unrefined experiments.

A typical problem that occurs when horizontally refined grids are used is the reflection and scattering of waves at the transition between coarse and fine parts of the grids. It is well known from classic papers that study the error caused by grid refinement in computational fluid dynamics [see e.g., Trefethen (1982) and Vichnevetsky (1987) for studies of hyperbolic equations] that small-scale waves with a wavelength close to the grid spacing can be affected strongly when simulations are performed on refined grids, while large-scale features typically appear to be rather unaffected. For linear equations of motion, dispersion relations and group velocities can be calculated for the considered discretization methods; this allows a detailed analysis of the behavior of waves at the transition zones between coarse and fine grids. Results indicate that, when the wavelength is of the same order of magnitude as the grid spacing, severe reflection and scattering occurs for almost every discretization scheme. These scatterings will also occur when refinement is applied in dynamical cores for geophysical applications (Ullrich and Jablonowski 2011; Long and Thuburn 2011). On the other hand, the first approaches using grids of variable horizontal resolution for three-dimensional models of atmosphere and ocean do not show fundamental problems in the dynamics at the mesh-transition zones (Skamarock et al. 2012; Ringler et al. 2013).

In this paper, we investigate the effect of grid refinement in a shallow-water model based on a hybrid finite-element discretization method that was especially developed for geophysical applications and from which we expect an excellent behavior on refined grids. This discrete model allows us to create a kind of "best-casescenario": if this model does not perform well on refined grids than other models will potentially experience even more severe problems. The hybrid finite-element combines a continuous second-order representation for the height field with a discontinuous first-order representation for the velocity field on a triangular grid-the $P_{1}^{\mathrm{DG}} P_{2}$ finite element (Cotter et al. 2009a; Düben et al. 2012). The element fulfills the Ladyzhenskaya-BabuskaBrezzi condition, which is a necessary condition for convergence in finite-element modeling, and was designed to represent the geostrophic balance at the same time (Cotter et al. 2009a,b). This is remarkable for a low-order finite element and necessary to avoid spurious modes in geophysical applications (Le Roux et al. 1998). These properties make the hybrid element a promising candidate to form the dynamical cores of future weather or climate models. In principle, the considered element allows $h$ and $r$ refinement (either new grid points are introduced into the domain, or given grid points are moved toward regions of specific interest), and has already been used on unstructured and refined grids in idealized applications (Comblen et al. 2010).

We provide a detailed investigation of the errors caused by the use of grid refinement in view of critical geophysical quantities for the specific $P_{1}^{\mathrm{DG}} P_{2}$ finiteelement setup. More precisely, we study the influence of grid refinement on the energy/enstrophy cascade, on the representation of boundary currents, on energy conservation, and on the ability to represent the geostrophic balance. The influence of the structure of the transition zone between coarse and fine parts of the grid and how the error behaves when the change of resolution is increased is tested as well.

An analytical study seems to be extremely difficult, if not impossible, for nonlinear and multidimensional equations in complex geometries. We therefore resort to an experimental approach and carry out several numerical experiments. We simulate idealized test cases such as a zonal geostrophic flow, wave packets with various wavelengths, two-dimensional turbulent cascades, a zonal flow over an isolated mountain, and Munk gyres. We perform these simulations on refined grids, where refinement is realized by introducing new grid points to the original grid (so-called $h$ refinement). Since it is known from the literature that pronounced errors occur when abrupt changes of the grid spacing are considered, we investigate sudden changes where grid resolution is increased by a factor of 2,4 , or 8 within one grid spacing of the unrefined grid.

We believe that such a study is relevant for many of the next generation ocean and atmosphere models that have a grid refinement capability. Examples are the hybrid finite-volume/finite-difference models such as the Model for Prediction Across Scales (MPAS; Ringler et al. 2011, 2013), or the icosahedral nonhydrostatic general circulation model (ICON; Bonaventura and 
Ringler 2005) and the finite-element models: the FiniteElement-Sea Ice-Ocean Model (FESOM; Danilov et al. 2004), the Community Atmosphere Model Spectral Element (CAM-SE; Taylor et al. 1997), or the Imperial College Ocean Model (ICOM; Piggott et al. 2008). There are also approaches that implement local zooming via nesting (see e.g., Harris and Durran 2010). A further step that is discussed especially in atmospheric science, is the use of adaptive grid refinement techniques [see Behrens (2006) for an overview].

The paper is organized as follows. In section 2, we give a brief overview about the model setup. In section 3, we present numerical results. In section 4 , we draw the conclusions.

\section{Model setup}

In this section we give a brief introduction to the used $P_{1}^{\mathrm{DG}} P_{2}$ finite-element model for the shallow-water equations. A detailed description of the model setup can be found in Düben et al. (2012).

\section{a. The viscous shallow-water equations}

We consider the viscous shallow-water equations in nonconservative form:

$$
\begin{gathered}
\partial_{t} \mathbf{u}+\mathbf{u} \cdot(\nabla \mathbf{u})+f \mathbf{k} \times \mathbf{u}+g \nabla h-\frac{1}{H} \nabla \cdot[H \nu(\nabla \mathbf{u})]=\boldsymbol{\tau} \\
\partial_{t} h+\nabla \cdot(H \mathbf{u})=0
\end{gathered}
$$

where $\mathbf{u}$ is the two-dimensional velocity vector, $f$ is the Coriolis parameter, $\mathbf{k}$ is the vertical unit vector, $g$ is the gravitational acceleration, $\nu$ is the eddy viscosity, $\boldsymbol{\tau}$ is a forcing term (e.g., wind in ocean applications), $h$ is the surface elevation, and $H$ is the height of the fluid column given by $H=h-h_{b}$, where $h_{b}$ is the bathymetry. The prognostic variables are the surface elevation and velocity.

\section{b. Discretization}

Following the typical finite-element approach, we expand the physical fields into sets of basis functions $N_{i}$ and $M_{i}$ :

$$
\mathbf{u}=\sum_{i=1}^{N_{u}} \mathbf{u}_{i} N_{i} \quad \text { and } \quad h=\sum_{i=1}^{N_{h}} h_{i} M_{i} .
$$

We use a $P_{1}^{\mathrm{DG}} P_{2}$ finite element to discretize the equations. This means that we employ discontinuous linear Lagrange polynomials for the representation of the velocity field $N_{i}$, and globally continuous quadratic
Lagrange polynomials for the representation of the height field $M_{i}$. Each triangular cell has three degrees of freedom for each component of velocity located at the vertices of the cells and six degrees of freedom for the height field located at the vertices and edges. While the degrees of freedom of the height field are shared with the surrounding cells, the degrees of freedom of the velocity field belong to a specific cell, which leads to a discontinuous representation.

Time integration is performed by an explicit threelevel Adams-Bashforth method. The equation

$$
\partial_{t} \boldsymbol{\psi}=R(\boldsymbol{\psi})
$$

where $R$ denotes the right-hand side of the system and $\psi$ is the vector of prognostic variables, is discretized in time by

$$
\boldsymbol{\psi}^{j+1}=\boldsymbol{\psi}^{j}+\Delta t\left[\frac{23}{12} R\left(\boldsymbol{\psi}^{j}\right)-\frac{4}{3} R\left(\boldsymbol{\psi}^{j-1}\right)+\frac{5}{12} R\left(\boldsymbol{\psi}^{j-2}\right)\right]
$$

where $\boldsymbol{\psi}^{j}$ is the vector of state variables at the $j$ th time step.

\section{c. Grids and grid refinement procedure}

In principle the model is applicable to each kind of triangular grid. In this publication, we use two types of standard grids on which refinement is performed. On the one hand, we use structured triangular grids that provide a uniform coverage of the plane. The grids are developed from rectangular grids by bisecting each rectangular into two triangles. On the other hand, we use icosahedral geodesic grids that provide a quasi-uniform coverage of the sphere (Baumgardner and Frederickson 1985).

In the $h$-refinement procedure which is used to refine the (quasi) uniform standard grids, an area of specific interest is specified where the original triangles are split into four new triangles (sketched in Fig. 1). The grids remain conformal and do not show hanging nodes. Changes in the grid spacing are always performed in an abrupt way, which means that the grid spacing is changing by a factor of 2,4 , or 8 within one grid spacing of the coarse grid. In the standard procedure the triangles in the transition zone between coarse and fine parts of a grid are split into two new triangles, as shown in Fig. 1. For global applications, the new vertices are projected onto the sphere.

\section{Numerical results}

In this section we present the results of five test cases that we have designed to understand the impact of static 

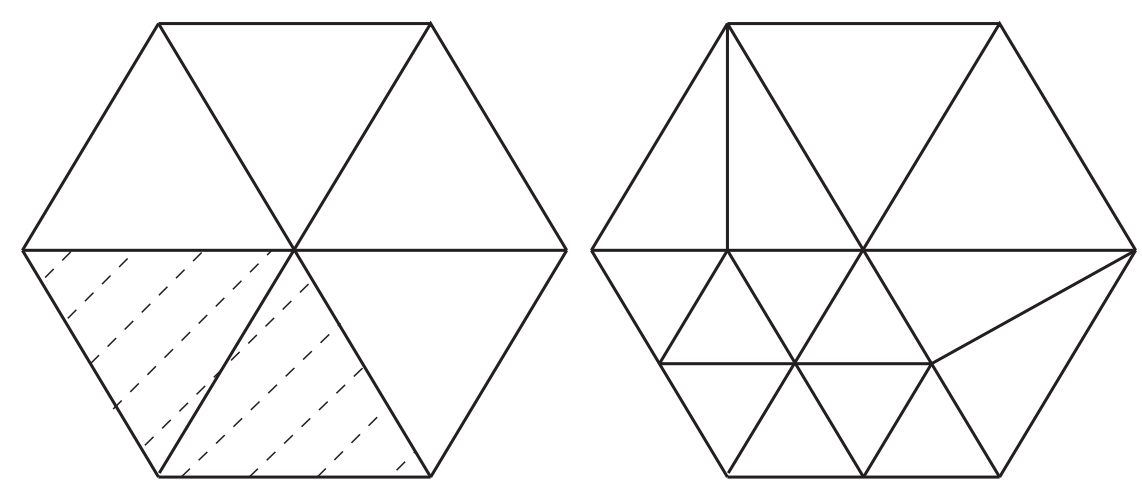

FIG. 1. Sketch of the refinement procedure. (left) An area of interest is specified in the original grid,

(right) the triangles are divided into four new triangles, and a transition zone is introduced.

local grid refinement. The first test (section 3a) uses a zonal geostrophic flow to evaluate the effect of grid refinement on the representation of geostrophic balance. In the second test (section 3b), we investigate the behavior of wave packets with variable wavelength at the transition zone of the grid. In the third test (section 3c) we consider randomly initialized physical fields to investigate the effect of grid refinement on turbulent decays. In tests four and five, we consider the influence of grid refinement in flows over topography (section 3d) and with lateral boundaries (section $3 \mathrm{e}$ ).

The following nomenclature is used to describe the varying grid resolution, for example, for a grid called $\mathrm{H}_{0} \mathrm{~T}_{1} \mathrm{R}_{1}$. The subscript of $\mathrm{H}$ indicates the coarsest resolution in the domain compared to the initial grid $\mathrm{H}_{0}$, the subscript of $\mathrm{T}$ indicates the type of the transition zone; $\mathrm{L}$ or $\mathrm{R}$ indicate whether the left or the right side was refined by the number of levels indicated by the subscript.

\section{a. Geostrophic balance affected by grid refinement}

In this subsection, we simulate a global steady-state zonal geostrophic flow. The test was proposed by Williamson et al. (Williamson et al. 1992, test case 2). The $P_{1}^{\mathrm{DG}} P_{2}$ element was designed carefully to satisfy the primary balances of the linear shallow-water equations without producing strong spurious modes and it was already tested that $P_{1}^{\mathrm{DG}} P_{2}$ is able to represent geostrophic balance properly for the linear shallow-water equations (Cotter et al. 2009a; Maddison et al. 2011). Here, we want to test if geostrophic balance is still represented properly when grid refinement is strongly changing the aspect ratio between adjacent grid cells, for the nonlinear equations on the sphere.

The simulated westerly flow has a maximum velocity of ca. $39 \mathrm{~m} \mathrm{~s}^{-1}$ and a fluid depth varying between 1000 and $3000 \mathrm{~m}$. It is a stationary solution of the nonlinear shallow-water equations. The flow is zonally symmetric. For this test case it is crucial that the model is starting from a balanced state. We initialize the finite-element model as follows: For the height field we use the prescribed values of the analytic initial conditions at the nodes of the continuous basis functions. For the corresponding velocity field we solve a system of equations to fit the discontinuous basis functions as far as possible to the steady state of the linear shallow-water equations, to assure balanced initial conditions.

We use three different grids for simulations. One uniform icosahedral grid with a typical edge length of about $480 \mathrm{~km}$, the same grid with six refinement regions that are circular in longitude-latitude space with a diameter of about $60^{\circ}$, and the same grid that has only the one refinement region at the equator (see Fig. 2). We used a time step of only $10 \mathrm{~s}$.

Figure 3 shows the pattern of the absolute error of the height field for different time steps and the three grids. After 5 days the wave- 5 grid imprint of the icosahedral grid is visible in the error plots for the uniform simulation. For the refined simulations with six circles refined, a wave-2 imprint dominates, which is caused by the anisotropy of the refinement zones (cf. Figs. 2 and 3). A change of the error pattern happens on time scales of days as can be seen from the small differences between days 5 and 5.5 .

To get a better understanding of the source of errors, Fig. 4 shows the $L_{1}$-error and $L_{2}$-error norm of the height field against time, the $L_{2}$ norm of the divergence field against time, the errors in energy conservation for the different grids against time, and the $L_{2}$ error for the height field at day 10 against the largest edge length. The error norms are defined as

$L_{1}(t)=\frac{\int_{\Omega}\left|h^{j}-h_{a}^{j}\right| d x}{\int_{\Omega}\left|h_{a}^{j}\right| d x}$, and $L_{2}(t)=\sqrt{\frac{\int_{\Omega}\left(h^{j}-h_{a}^{j}\right)^{2} d x}{\int_{\Omega}\left(h_{a}^{j}\right)^{2} d x}}$, 


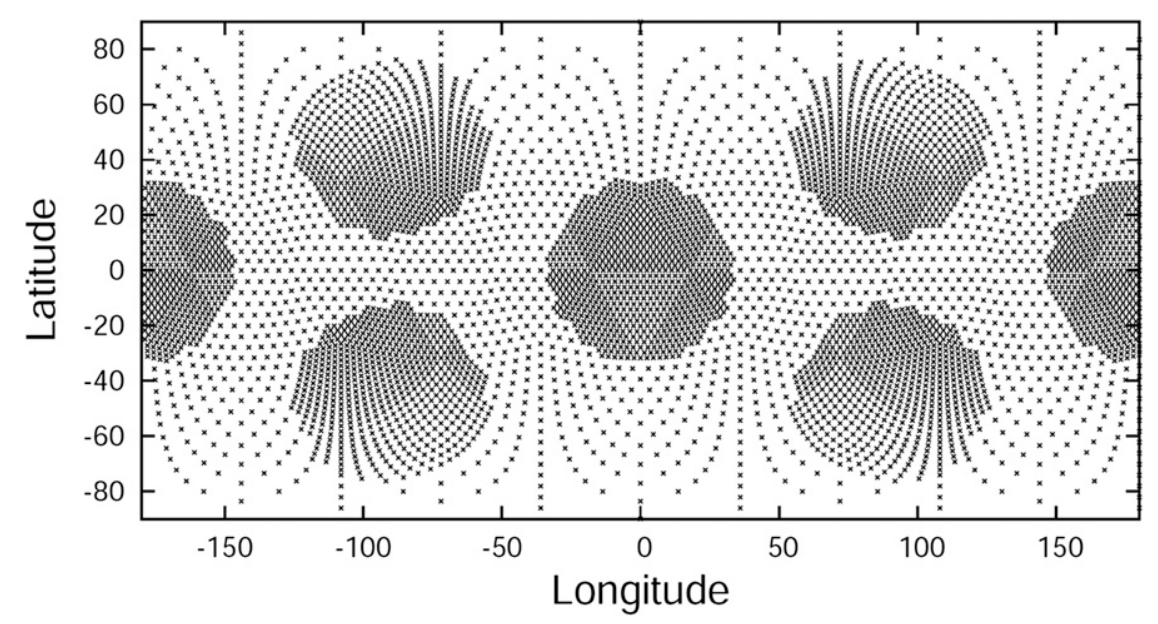

FIG. 2. Geostrophic balance affected by grid refinement: positions of the vertices in the grid with six refinement zones. A second simulation has only the one refined circle in the center and a third simulation is using the unrefined grid.

where $h^{j}$ is the computed height at time step $j$ compared to the analytical solution $h_{a}^{j}$. Here $\Omega$ indicates that we integrate over the whole domain. It is interesting that the simulation with only one refined circle shows the highest local and global errors (see Figs. 3 and 4).

Since flows in geostrophic balance have vanishing divergence, the $L_{2}$ norm of the velocity divergence can serve as an indicator for a violation of the geostrophic balance. From Fig. 4 we infer that for any of the simulations the $L_{2}$ error of the divergence shows no significant tendency to increase over the simulation length of 50 days. We therefore attribute the existing errors rather to the broken zonal symmetry of the flow than to the inability of the $P_{1}^{\mathrm{DG}} P_{2}$ model to maintain geostrophic balance on refined grids.

Regarding energy conservation we remark that the considered discrete shallow-water model does not strictly conserve energy. The procedure to calculate the relative error for energy is described in section 5 in the appendix. Figure 4 shows that the error in energy conservation is getting smaller when the refined area is increased.

It was found in Düben et al. (2012) that the given model shows third-order convergence with uniform resolution for the Williamson test case 2, although a second-order convergence would be expected because of the linear representation of velocity. This can be explained since Cotter and Ham (2011) showed in a Helmholtz decomposition for the linear shallow-water equations that the used $P_{1}^{\mathrm{DG}} P_{2}$ element exhibits thirdorder convergence rates for Rossby waves (at least with $\beta$-plane approximation). The errors caused by zonal asymmetry will propagate as Rossby waves. It is visible in Fig. 4 that this third-order convergence breaks down when refined grids are used. However, second-order convergence is maintained.

We summarize the results of this section by concluding that the grid refinement perturbs the test case slightly due to a break of zonal symmetry, while the higher accuracy within the refinement region reduces the error locally. The reduction of the local error due to higher resolution is able to level out the error due to the break of zonal symmetry on the grid with six refined circles, but not on the grid with only one refined circle. Geostrophic balance is maintained.

\section{b. Wave propagation affected by grid refinement}

In this subsection we study the transition of wave packets between coarse and fine areas of a grid. We simulate waves with four different wavelength that travel on grids with an abrupt change in resolution and evaluate two different types of transition zones and two different angles the incoming waves form with the change in resolution. The model results are evaluated with regard to the error in energy conservation, and the change of the spectra of kinetic energy.

The wave packets are initialized as follows:

$$
\begin{aligned}
& u=u_{a} \exp \left[-\frac{\left(x-x_{0}\right)^{2}}{2 \sigma^{2}}\right] \sin \left[\frac{2 \pi n\left(x-x_{0}\right)}{L_{x}}\right], \quad v=0, \quad \text { and } \\
& h=\frac{u_{g}^{2}}{g}+\frac{u_{a} \times u_{g}}{g} \exp \left[-\frac{\left(x-x_{0}\right)^{2}}{2 \sigma^{2}}\right] \sin \left[\frac{2 \pi n\left(x-x_{0}\right)}{L_{x}}\right],
\end{aligned}
$$

where $u_{a}$ is the maximal absolute value of the zonal velocity, $u_{g}$ is the group velocity of the wave packet, $\sigma$ 

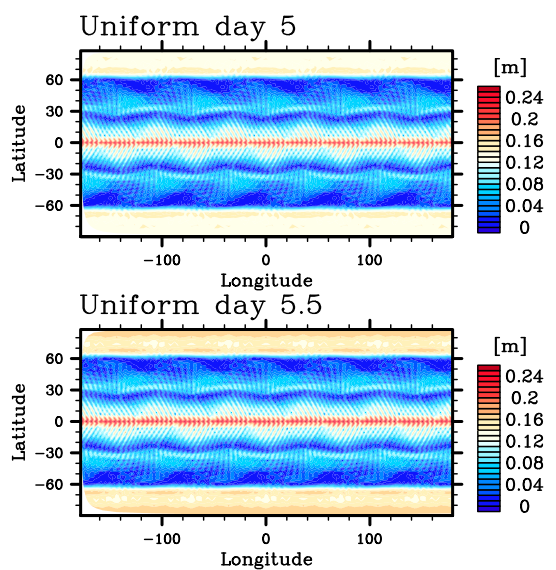

Uniform day 10

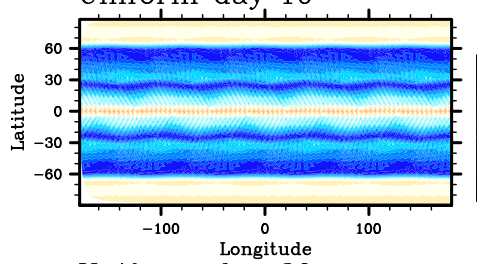

Uniform day 20

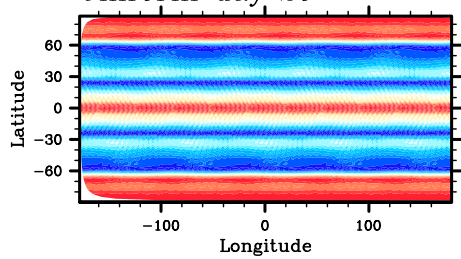

Uniform day 30

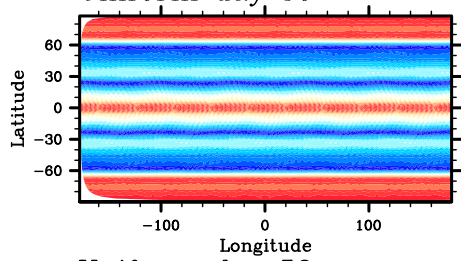

Uniform day 50
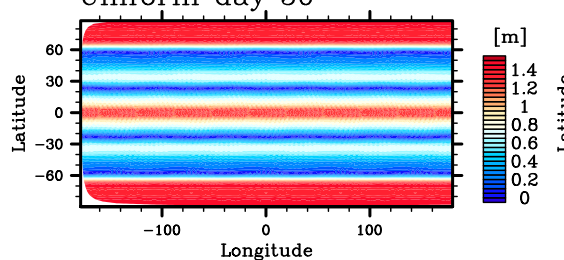
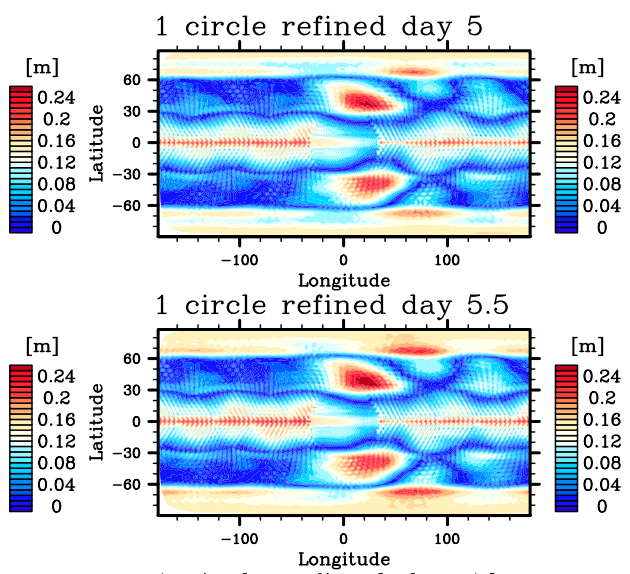

1 circle refined day 5.5
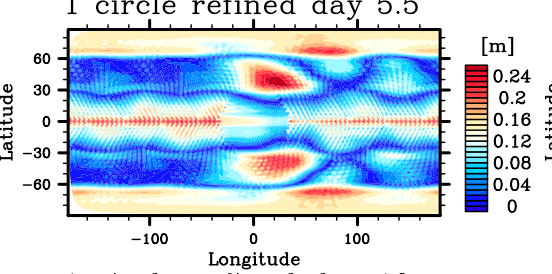

1 circle refined day 10
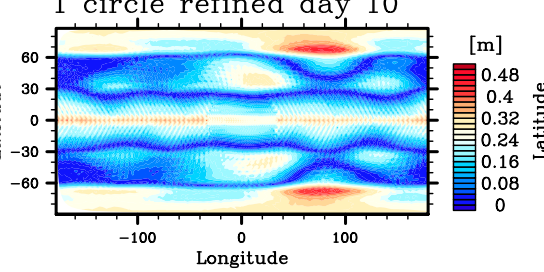

1 circle refined day 20

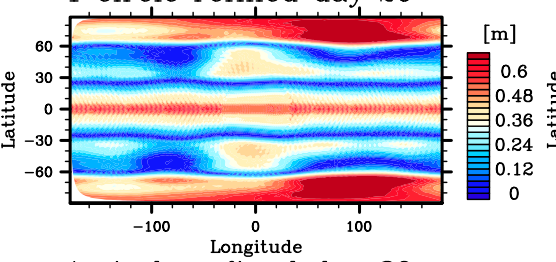

1 circle refined day 30
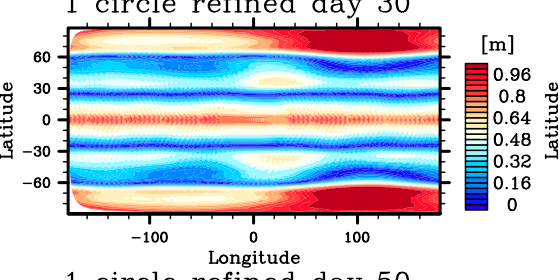

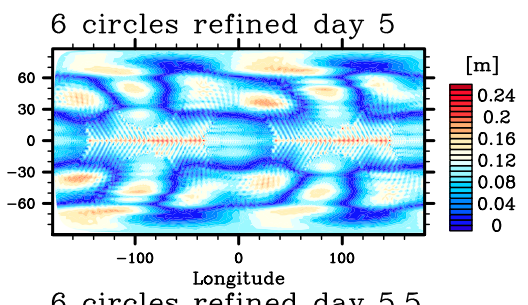

6 circles refined day 5.5

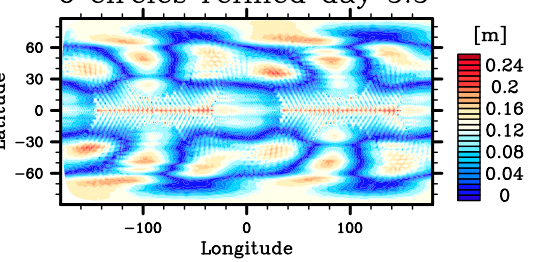

6 circles refined day 10

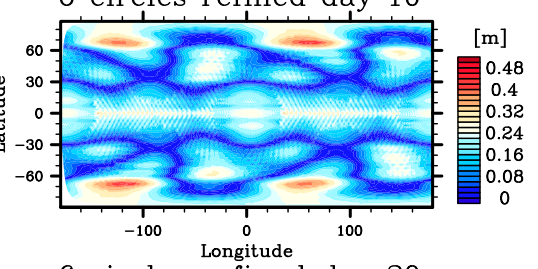

6 circles refined day 20

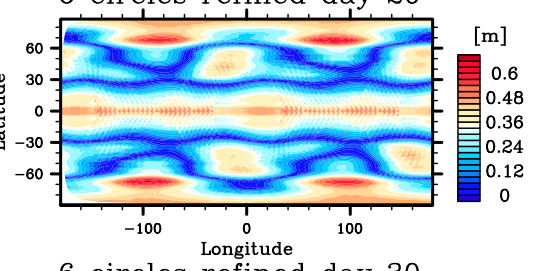

6 circles refined day 30

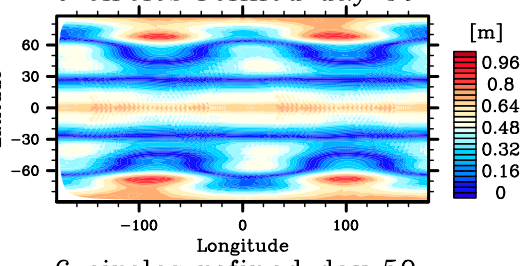

6 circles refined day 50
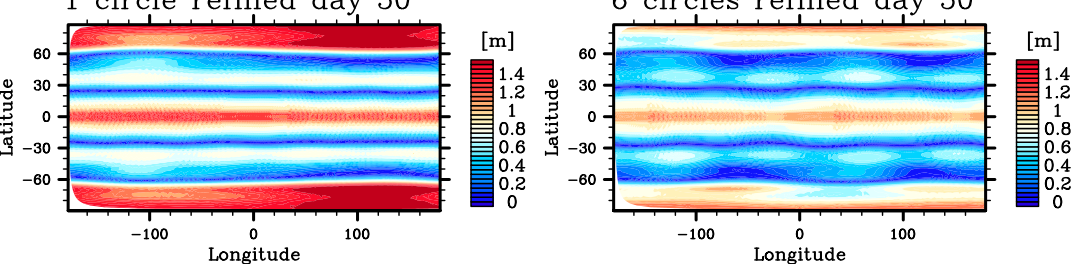

FIG. 3. Geostrophic balance affected by grid refinement: absolute error for the height field (from top to bottom) at days 5, 5.5, 10, 20, 30, and 50 on the (left) uniform and the grids with (middle) one and (right) six refined circles (cf. Fig. 2). Note the changed color bar for the different rows. The wave- 5 and -2 patterns are clearly visible for the unrefined case and the case with six refined circles, due to the symmetry of the grids. The case with only one refinement zone shows the highest local errors.

is the standard deviation that adjusts the width of the packet, $x_{0}$ is the center of the packet, and $n$ is the wavenumber that indicates the ratio how often the wavelength of the inner frequency of the packet fits into the length of the domain $L_{x}$. Simulations are performed on a plane with periodic boundary conditions.
As initial values we used the following: $u_{a}=2.0 \mathrm{~m} \mathrm{~s}^{-1}$, $u_{g}=250.0 \mathrm{~m} \mathrm{~s}^{-1}, \sigma^{2}=0.006 L_{x}^{2}, L_{x}=10^{7} \mathrm{~m}, L_{y}=1.6 \times$ $10^{6} \mathrm{~m}$, and $x_{0}=0.25 \times L_{x}$. Initially, the wave packet consists of linear gravity waves and is located in the left half of the domain. We simulate the inviscid nonlinear shallow-water equations without Coriolis force [Eqs. (1) and (2) with $f=\nu=0$ ]. We use a time step of $10 \mathrm{~s}$. 

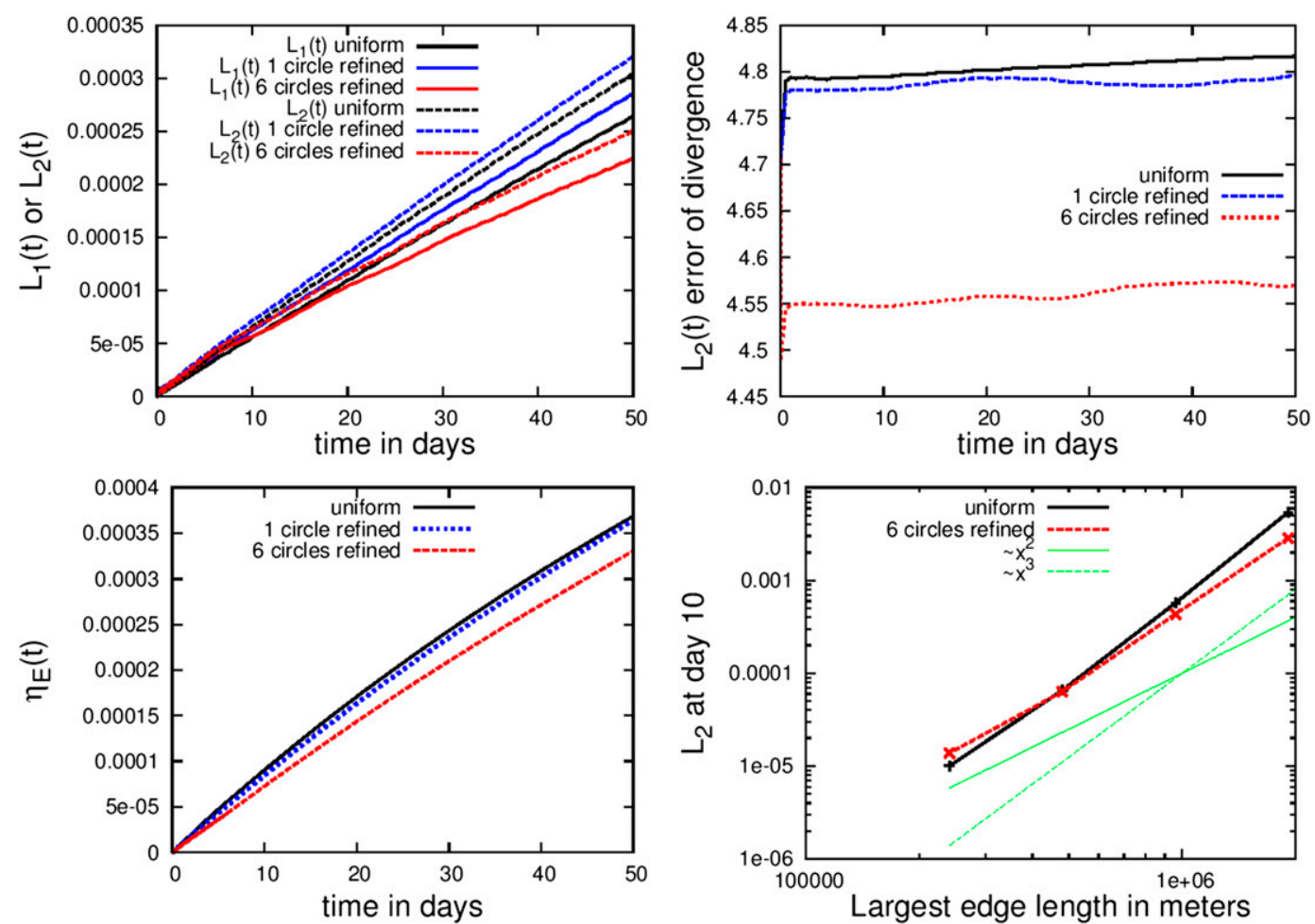

FIG. 4. Geostrophic balance affected by grid refinement: (top left) $L_{1}$-error and $L_{2}$-error norm of the height field against time, (top right) $L_{2}$ norm of the divergence field against time, (bottom left) error in energy conservation for the different grids, and (bottom right) $L_{2}$-error norm of the height field against the largest edge length.

We use a grid that consists of $50 \times 8$ squares, each of them divided into two triangles, as the unrefined starting point for grid refinement $\left(\mathrm{H}_{0}\right)$. We refine either the left or the right half of the domain, and increase the resolution by a factor of 2,4 , or 8 . We use two different types of transition zones between the refined and unrefined areas. Both of them lead to an abrupt change in resolution. The transition zones of the grids are sketched in Fig. 5. In the first type the triangles at the transition zone do not have steep angles $\left(\mathrm{H}_{0} \mathrm{~T}_{1} \mathrm{R}_{1}, \mathrm{H}_{0} \mathrm{~T}_{1} \mathrm{R}_{2}\right)$. In the second type, the triangles at the transition show steep angles $\left(\mathrm{H}_{0} \mathrm{~T}_{2} \mathrm{R}_{1}, \mathrm{H}_{0} \mathrm{~T}_{2} \mathrm{R}_{2}, \mathrm{H}_{0} \mathrm{~T}_{2} \mathrm{R}_{3}\right.$; there are angles with less than $6^{\circ}$ ). Additionally to the grids in Fig. 5 we use grids in which the left half of the domain is refined $\left(\mathrm{H}_{0} \mathrm{~T}_{1} \mathrm{~L}_{1}, \mathrm{H}_{0} \mathrm{~T}_{1} \mathrm{~L}_{2}, \mathrm{H}_{0} \mathrm{~T}_{2} \mathrm{~L}_{1}, \mathrm{H}_{0} \mathrm{~T}_{2} \mathrm{~L}_{2}, \mathrm{H}_{0} \mathrm{~T}_{2} \mathrm{~L}_{3}\right)$, and uniformly refined grids in which the whole domain offers doubled or 4 times the resolution of the coarse grid $\left(\mathrm{H}_{1}, \mathrm{H}_{2}\right)$.
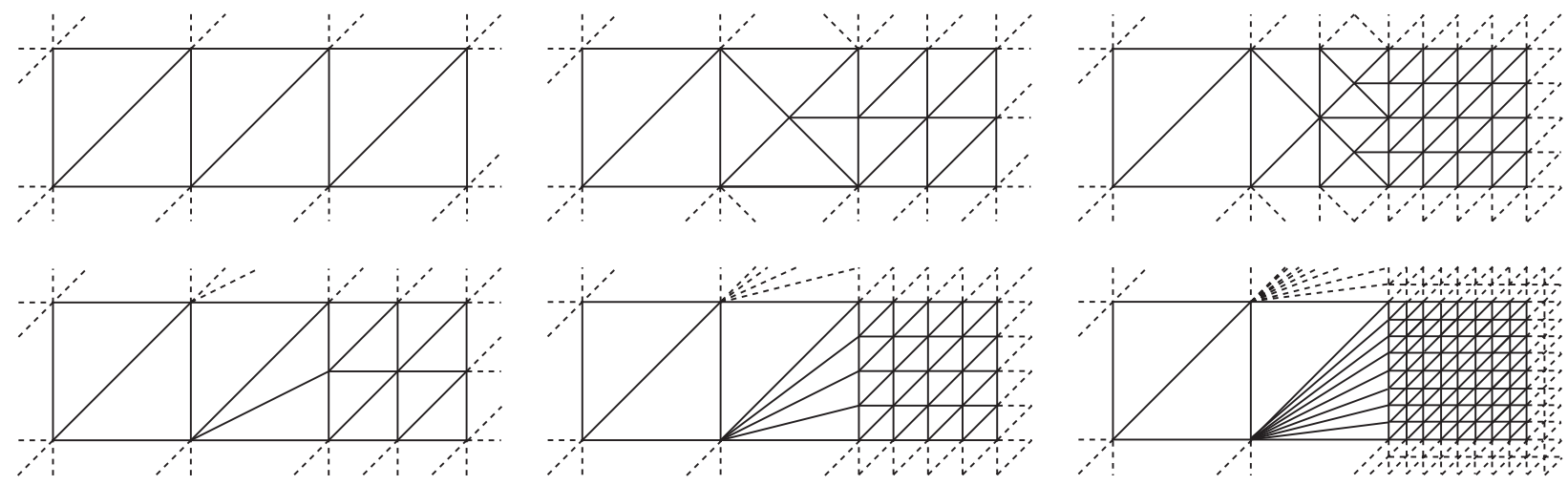

FIG. 5. Wave propagation affected by grid refinement: transition zone between the left- and the right-hand side of the grids. (top left) $\mathrm{H}_{0}$, (top middle) $\mathrm{H}_{0} \mathrm{~T}_{1} \mathrm{R}_{1}$, (top right) $\mathrm{H}_{0} \mathrm{~T}_{1} \mathrm{R}_{2}$, (bottom left) $\mathrm{H}_{0} \mathrm{~T}_{2} \mathrm{R}_{1}$, (bottom middle) $\mathrm{H}_{0} \mathrm{~T}_{2} \mathrm{R}_{2}$, and (bottom right) $\mathrm{H}_{0} \mathrm{~T}_{2} \mathrm{R}_{3}$. Additional grids were used with the left-hand side of the grid refined. 

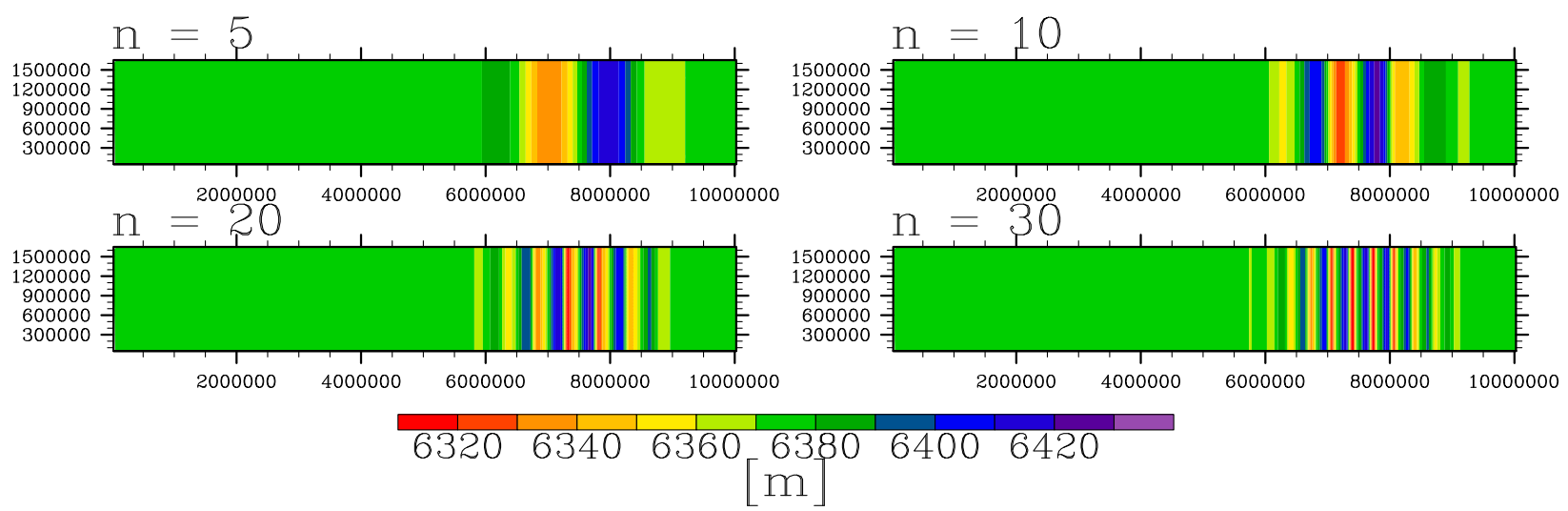

FIG. 6. Wave propagation affected by grid refinement: height field after $20000 \mathrm{~s}$ for (from top left to bottom right) $n=5, n=10, n=20$, and $n=30$, for simulations on the finest uniform grid $\left(\mathrm{H}_{2}\right)$. The wave packet is represented properly.

At least two grid points are necessary to resolve a given wavelength in a gridpoint finite-difference model. The number of cells by which one wavelength of the internal wave is resolved on the coarse grid $\mathrm{H}_{0}$ is given by $50 / n$. For our simulations, we consider two wave packets where the internal wave should be resolved properly ( $n=5$ and $n=10)$, and two wave packets where the coarse resolution is very close to, or below the possible minimum to resolve the wave packet $(n=20$ and $n=30$ ).

Figure 6 shows the height field of the reference runs on the finest uniform grid $\left(\mathrm{H}_{2}\right)$ after $20000 \mathrm{~s}$. The specific time step is chosen such that the wave packet has crossed the transition zone once, and traveled for the same time on each side of the grid. Figure 7 shows the spectra of kinetic energy for the simulation with $n=20$. The procedure to calculate the spectral coefficients is described in the appendix. All spectral coefficients $E_{i j}$ are zero for $j$ different from zero, since the physical fields only vary in the $x$ and not in the $y$ direction. The kinetic energy spectrum of the initial wave packet has the form of a Gaussian distribution centered around $n$. While there is no visible difference between the height field of the wavepacket at initialization (not shown here) and after $20000 \mathrm{~s}$, the initial Gaussian distribution is slowly getting weaker with increasing time, and additional Gaussian distributions form at multiples of the wavenumber $n$, since advection induces weak scale interactions.

Figure 8 shows the height field after 20000 s for simulations on the $\mathrm{H}_{0} \mathrm{~T}_{2} \mathrm{~L}_{3}$ and the $\mathrm{H}_{0} \mathrm{~T}_{2} \mathrm{R}_{3}$ grid. Out of the 13 grids used, these are the grids for which we expect the largest errors, since they show the strongest change in resolution and triangles that are very narrow with very steep angles. As expected, differences between the grids are very small for the sufficiently resolved waves with $n=5$ and $n=10$ (less than $0.2 \mathrm{~m}$ for $n=5$ and less than $6 \mathrm{~m}$ for $n=10$ ), but they become clearly visible for higher values of $n$. Plots for the model runs on grids with lower refinement level (not shown here) look very similar to the runs on the $\mathrm{H}_{0} \mathrm{~T}_{2} \mathrm{~L}_{3}$ and the $\mathrm{H}_{0} \mathrm{~T}_{2} \mathrm{R}_{3}$ grid.

A consideration of the spectra of kinetic energy could give a better impression of the errors caused by grid refinement. To calculate the spectra a discrete Fourier transformation of the physical fields is needed, which is only possible for equidistant grids (see the appendix) and a locally refined grid is not equidistant by definition. To get at least an approximation of the spectra, we calculate discrete Fourier transformations using only the equidistant grid points of the initial, unrefined grids. To this end, the values of the discontinuous velocity are averaged at the vertices. The major shortcoming of this procedure is that the obtained spectra cover only wavelengths down to the doubled grid spacing of the

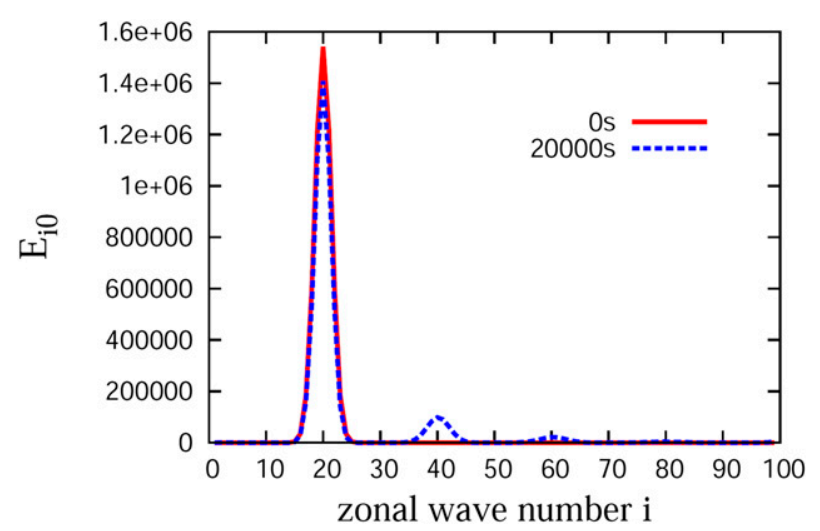

FIG. 7. Wave propagation affected by grid refinement: kinetic energy spectra calculated at initialization and after $20000 \mathrm{~s}$ for $n=$ 20 and a simulation on the finest uniform grid $\left(\mathrm{H}_{2}\right)$. The spectra are calculated by evaluating all of the $200 \times 64$ vertices of the grid, for the discrete Fourier transformations. The initial Gaussian distribution is slowly getting weaker with increasing time, and additional Gaussian distributions form at multiples of the wavenumber $n$, since advection induces weak scale interactions. 

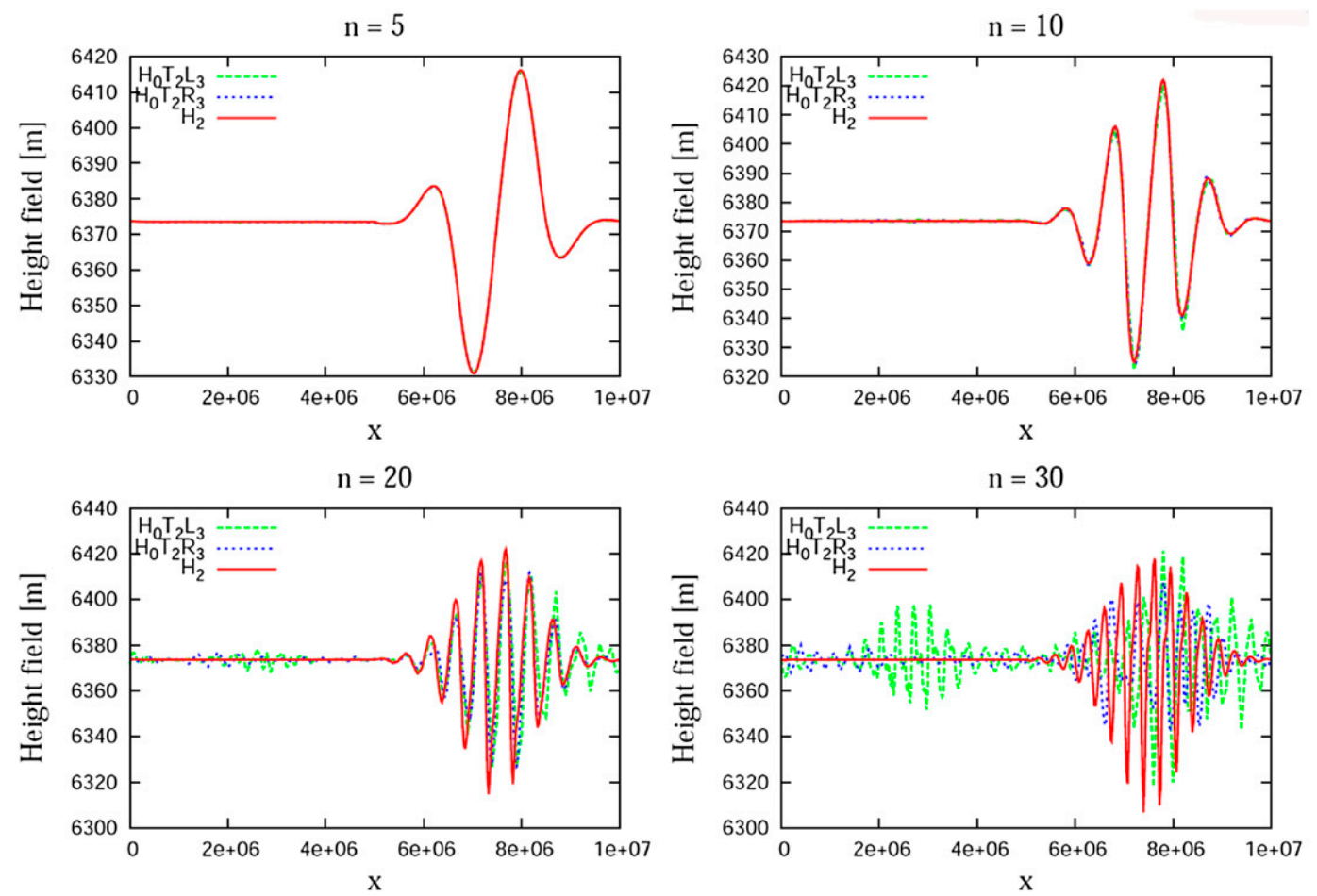

FIG. 8. Wave propagation affected by grid refinement: height field after $20000 \mathrm{~s}$ on the reference high-resolution grid $\left(\mathrm{H}_{2}\right)$ and the worst grids used $\left(\mathrm{H}_{0} \mathrm{~T}_{2} \mathrm{~L}_{3}\right.$ and $\left.\mathrm{H}_{0} \mathrm{~T}_{2} \mathrm{R}_{3}\right)$, for (from top left to bottom right) $n=5, n=10, n=20$, and $n=30$. The plots show the height field against the $x$ direction on different grids. Note that the simulation is still simulated with two horizontal dimensions.

initial, unrefined grid. It has to be kept in mind that this spectrum calculation does only yield a crude approximation when we interpret the results. When calculating the spectra on a mesh with the uniform grid spacing of the fine grid, the insufficient representation of the wave packet by the linear basis function for velocity in the coarse part perturbs the spectra calculated on the full grid. Therefore, this is not an option.

Figure 9 shows the approximated kinetic energy spectra after $20000 \mathrm{~s}$ for different values of the wavenumber $n$ for the different grids. We note that changes of the total level of the kinetic energy spectra do not indicate a change of total energy within the simulation. Energy can be transferred into potential energy or higher wavenumbers that are not covered by the approximated spectra.

For $n=5$ and $n=10$ the spectra are nicely separated according to the color code, meaning that we get three different results for uniform grids, grids for which the left-hand side is refined, and grids for which the righthand side is refined. It is surprising that differences for the different types of the transition zone and for grids with different changes of resolution are hardly visible and also that the spectra show clear differences for $n=$ 10 , while changes of the height field appear to be small (see Fig. 8). The same separation is still visible for $n=$ 20 , although the coarsest uniform grid $\left(\mathrm{H}_{0}\right)$ differs from the other uniform grids, since the wave packet is hardly resolved. Although most of the structure in the spectra is due to aliasing for $n=30$, it is obvious that the model fails to represent the wave packet on the coarsest uniform and the refined grids.

Figure 10 shows the relative error for energy for the different grids used. For the grids in which the righthand side is refined (blue lines), the error follows the $\mathrm{H}_{0}$ run, till the wave packets reach the transition zone. Afterward, the error in energy conservation strengthen the conclusion from Fig. 9 since errors depend only little on the refinement level (with a difference for the relative error of less than $1.0 \times 10^{-9}$ for $n=5$ after $20000 \mathrm{~s}$ ), and marginally on the type of the transition zone (with a difference of less than $1.5 \times 10^{-10}$ for $n=5$ after 20000 s). For the grids in which the left-hand side is refined (green lines), the errors follow the uniform model runs with corresponding resolution, till the wave packets reach the transition zone. Again, the errors differ only marginally for the different types of the transition zone (less than $2.0 \times 10^{-10}$ for $n=5$ ). Ignoring the model runs on the $\mathrm{H}_{0} \mathrm{~T}_{2} \mathrm{~L}_{3}$ and the $\mathrm{H}_{0} \mathrm{~T}_{2} \mathrm{R}_{3}$ grid, the relative errors on the different grids lie in between the relative errors of 

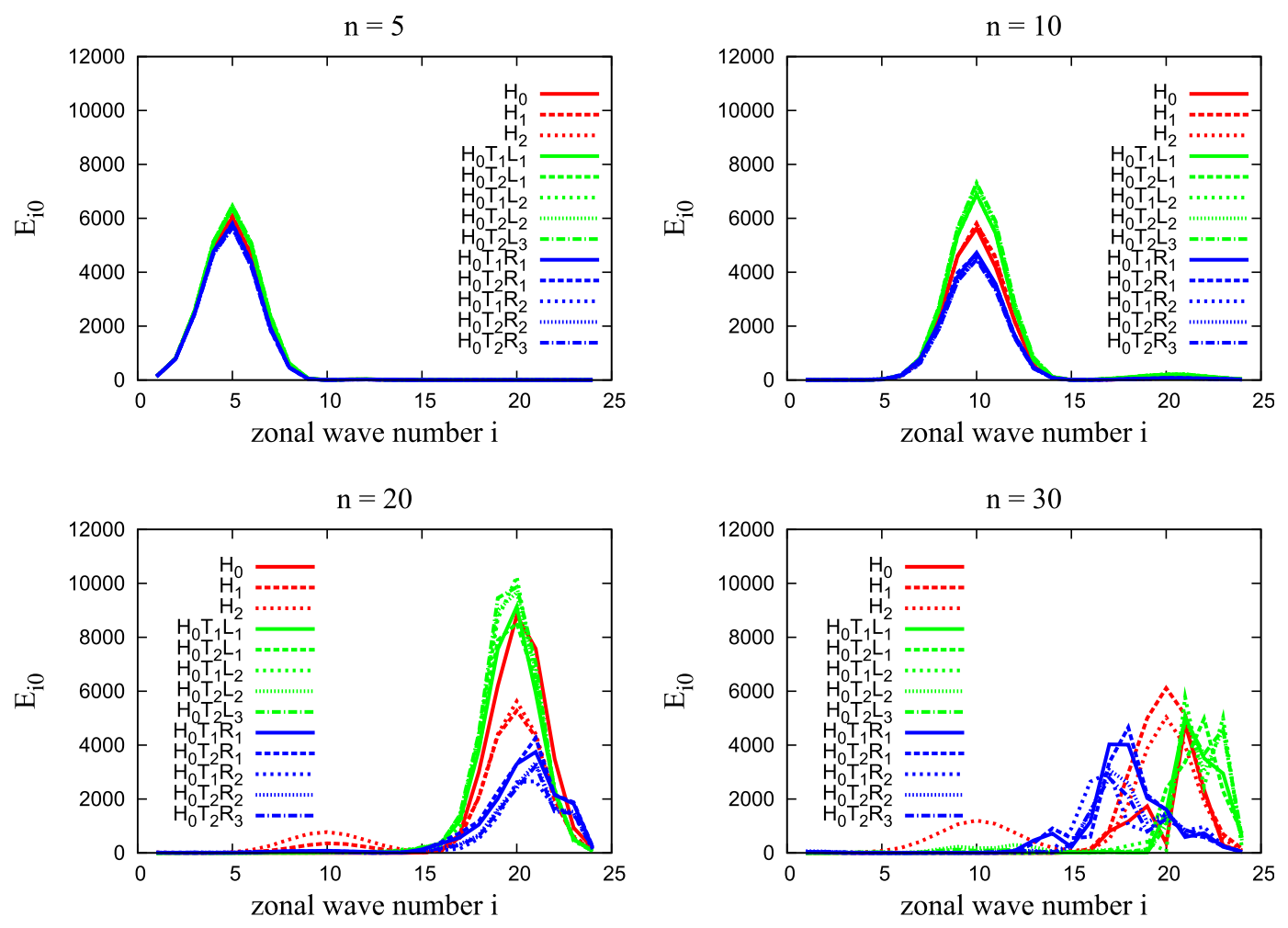

FIG. 9. Wave propagation affected by grid refinement: approximated kinetic energy spectra after $20000 \mathrm{~s}$ for (from top left to bottom right) $n=5, n=10, n=20$, and $n=30$. The spectra are calculated evaluating all of the $50 \times 8$ vertices of the coarsest uniform grid, for the discrete Fourier transformations. While the differences between different transition zones and different changes in resolution are marginal, the direction of the resolution change is important (coarse $\rightarrow$ fine or fine $\rightarrow$ coarse, see the color code). Details on the grids can be found in Fig. 5 .

the runs on the finest and coarsest uniform grids, for the cases where the wave packet is sufficiently resolved $(n=$ 5 and $n=10$ ). The model runs on the $\mathrm{H}_{0} \mathrm{~T}_{2} \mathrm{~L}_{3}$ and the $\mathrm{H}_{0} \mathrm{~T}_{2} \mathrm{R}_{3}$ grid, that show the highest change in resolution and the steepest angles, differ from the other runs for large wavelength $(n=5$ and $n=10)$.

To test the influence of the angle a wave forms with the transition zone, we divide the rectangular domain by a diagonal, where either the bottom-left $\left(\mathrm{H}_{0} \mathrm{~L}_{1} \mathrm{diag}\right)$, or the top-right $\left(\mathrm{H}_{0} \mathrm{R}_{1} \mathrm{diag}\right)$ part of the grid is refined by one refinement level, increasing the resolution by a factor of 2 (see Fig. 11). Figure 12 shows the approximated energy spectra of model runs on the two refined, and the uniform $\mathrm{H}_{1}$ grid. Results are similar as for the runs where either the left or the right half of the domain is refined. The spectra are separated by the side of the grid that has been refined.

The main results of this section are that differences for different types of transition zones and for grids with different changes in resolution are fairly small, and that changes of spectra can be strong although errors in the physical fields are hardly visible. Grid refinement has an influence on energy conservation, but the relative error is typically getting smaller compared to the unrefined simulation.

\section{c. Turbulent decay affected by grid refinement}

In this section, we investigate the influence of grid refinement on turbulent decays. The results of the previous section show that small-scale waves can be disturbed at transition zones between coarse and fine grids, even if changes appear to be fairly small when looking solely at the physical fields (cf. e.g., Figs. 8 and 9 for $n=$ 10). It is not obvious if the errors in wave propagation can influence isotropic turbulent cascades for which waves hit the boundary between the coarse and the fine part of the grid with all different wavelengths and angles.

We study a test case in which the physical fields are initialized randomly on a plane. The test is typically used to evaluate the cascade of kinetic energy in turbulent two-dimensional flows (McWilliams 2006), or for model evaluation (Ringler and Randall 2002; Bonaventura and Ringler 2005). In two-dimensional turbulent flows, energy is transferred from smaller to larger scales. The kinetic energy spectra should show a $n^{-3}$ cascade with the wavenumber (Salmon 1998). 

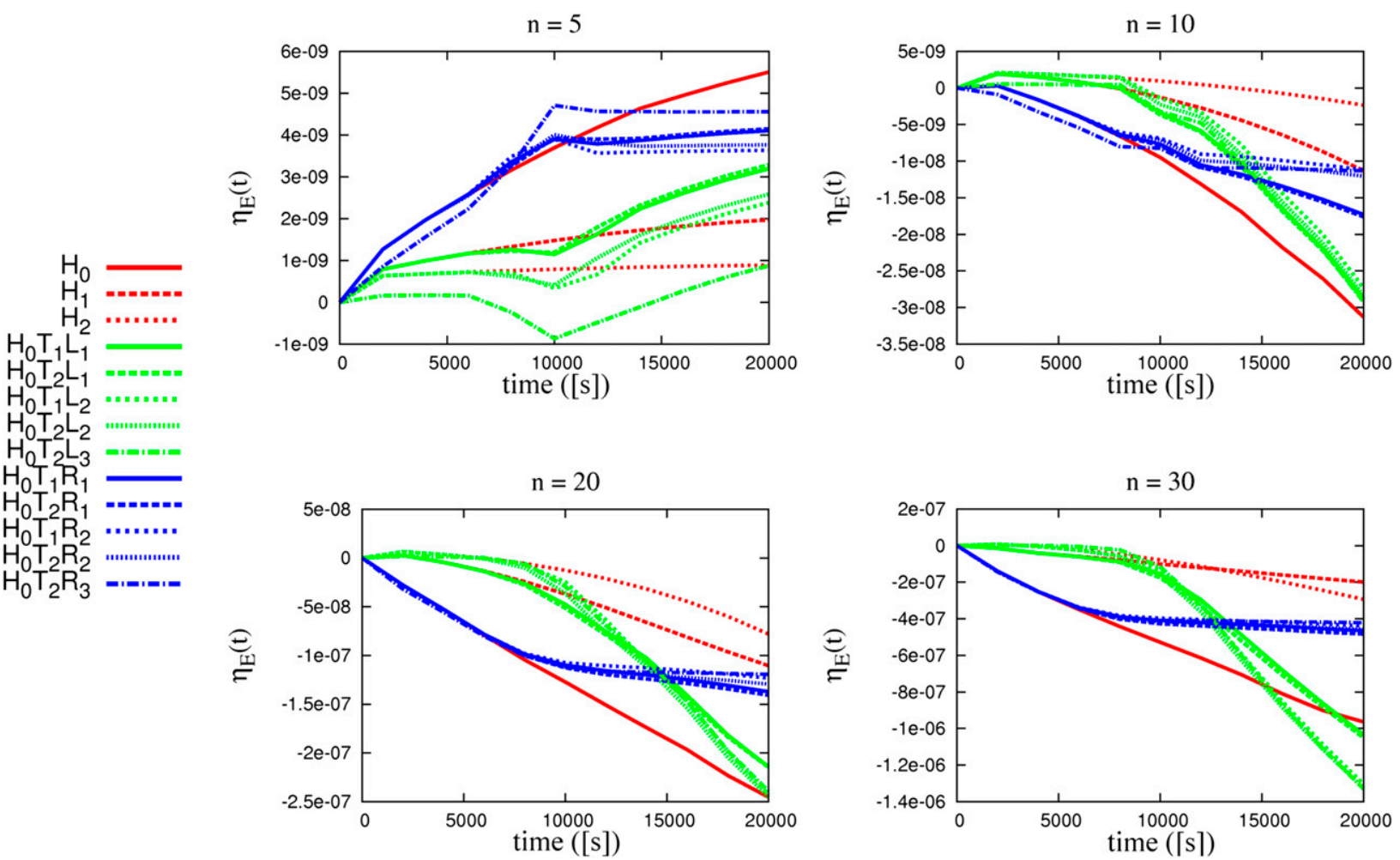

FIG. 10. Wave propagation affected by grid refinement: time evolution of the relative error for energy conservation for (from top left to bottom right) $n=5, n=10, n=20$, and $n=30$. In most cases, the relative errors on the refined grids are enclosed between the errors on the coarsest and finest uniform grid $\left(\mathrm{H}_{0}\right.$ and $\left.\mathrm{H}_{2}\right)$. The shape of the transition zone and the change in resolution seem to be rather unimportant. Details on the grids can be found in Fig. 5.

We use a grid that consists of $60 \times 60$ squares, each of them divided into two triangles, as a coarse uniform grid $\left(\mathrm{H}_{0}\right)$ for a periodic domain. We refine the $\mathrm{H}_{0}$ grid on the whole domain to obtain a second uniform grid with doubled resolution $\left(\mathrm{H}_{1}\right)$. Furthermore, we build up two grids in which the resolution in the right-hand side of the $\mathrm{H}_{0}$ grid is increased by a factor of 2 or $4\left(\mathrm{H}_{0} \mathrm{R}_{1}\right.$ and $\mathrm{H}_{0} \mathrm{R}_{2}$ ). The grids are plotted in Fig. 13. The transition zone between the coarse and fine part of the grid is the same as for the $H_{0} T_{1} R_{1}$ and the $H_{0} T_{1} R_{2}$ grid, plotted in Fig. 5.

We want to initialize the turbulent decay with white noise in the physical fields on the coarsest grid. To apply the same "random" initial conditions on each grid we describe the white noise as a superposition of all wave modes that are possibly described on the $\mathrm{H}_{0}$ grid with the same amplitude, using modes that are sinus shaped in the two spatial directions with wavelengths between

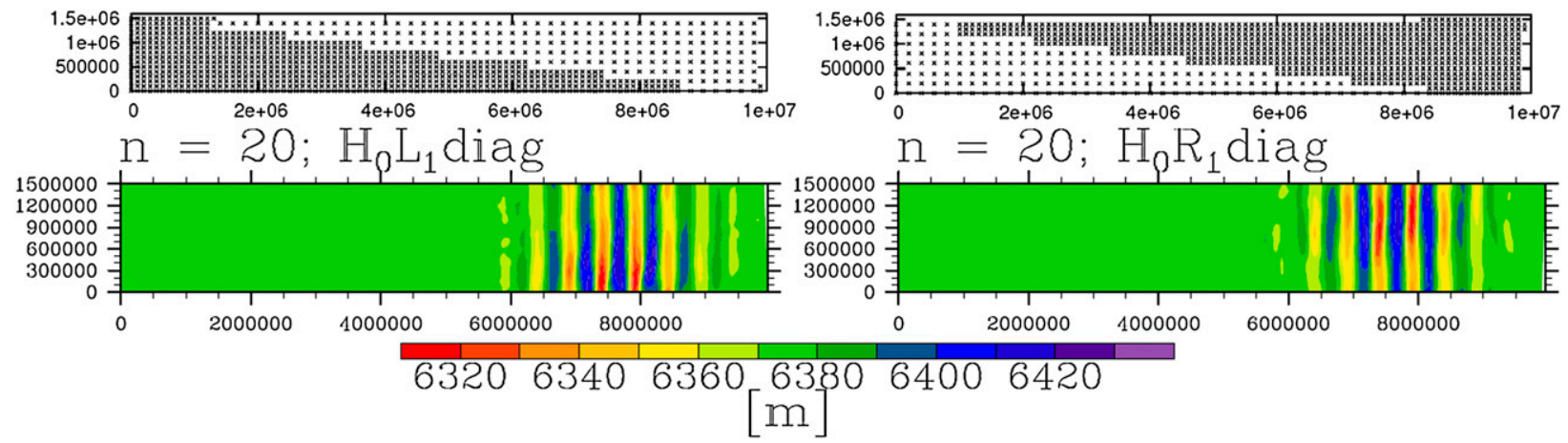

FIG. 11. Wave propagation affected by grid refinement: positions of the vertices in the (top left) $H_{0} L_{1}$ diag and (top right) the $H_{0} R_{1}$ diag grid, and height field after $20000 \mathrm{~s}$ for $n=20$ on the (bottom left) $\mathrm{H}_{0} \mathrm{~L}_{1}$ diag and the (bottom right) $\mathrm{H}_{0} \mathrm{R}_{1}$ diag grid. 


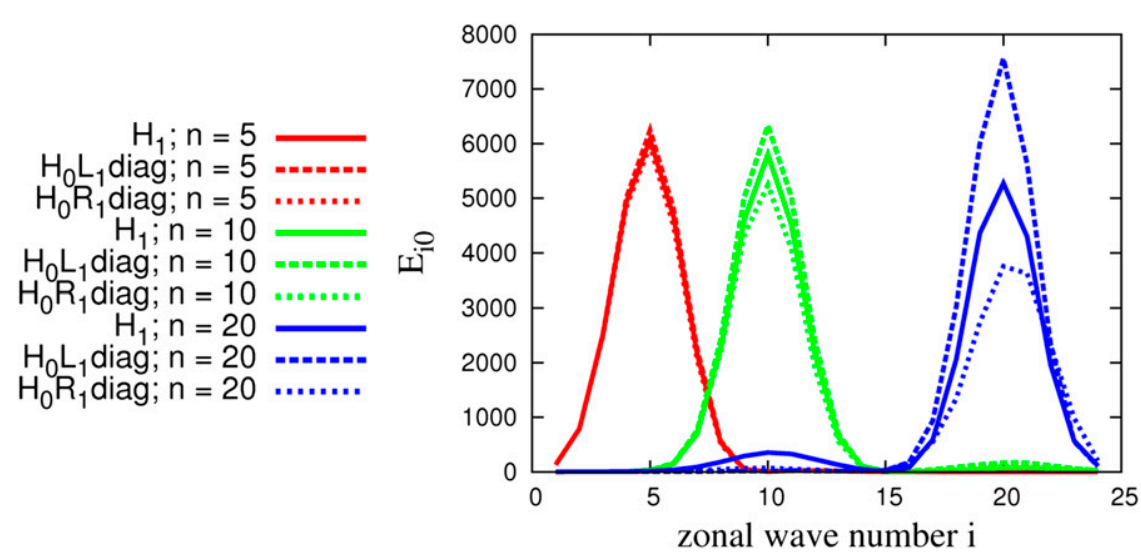

FIG. 12. Wave propagation affected by grid refinement: test on diagonally refined grids. Approximated kinetic energy spectra after $20000 \mathrm{~s}$ for different wavenumbers. The spectra are calculated evaluating all of the $50 \times 8$ vertices of the coarsest uniform grid, for the discrete Fourier transformations. The qualitative results are as in Fig. 9.

$2 \Delta x$ on the $\mathrm{H}_{0}$ grid and half the size of the domain. The initial conditions can be described as

$$
\begin{aligned}
\phi(x, y)= & \phi_{0}+\sum_{i=1}^{30} \sum_{j=1}^{30} \phi_{1} \sin \left(\frac{2 \pi i x}{L_{x}}+r_{1, i}\right) \sin \left(\frac{2 \pi j y}{L_{y}}+r_{2, j}\right) \\
& +\sum_{i=1}^{30} \phi_{1} \sin \left(\frac{2 \pi i x}{L_{x}}+r_{1, i}\right) \\
& +\sum_{j=1}^{30} \phi_{1} \sin \left(\frac{2 \pi j y}{L_{y}}+r_{2, j}\right)
\end{aligned}
$$

where $\phi$ represents either a component of velocity or height, $\phi_{0}$ and $\phi_{1}$ are constants associated with the physical field, and $r_{1, i}$ and $r_{2, j}$ are random numbers that are equally distributed between zero and $2 \pi$. As initial values we used the following: $u_{0}=0.0 \mathrm{~m} \mathrm{~s}^{-1}, u_{1}=$ $0.01 \mathrm{~m} \mathrm{~s}^{-1}, v_{0}=0.0 \mathrm{~m} \mathrm{~s}^{-1}, v_{1}=0.01 \mathrm{~m} \mathrm{~s}^{-1}, h_{0}=400.0 \mathrm{~m}$, and $h_{1}=1.0 \mathrm{~m}$. They result in absolute values of the velocity field that vary between 0.0 and $0.5 \mathrm{~m} \mathrm{~s}^{-1}$, and a fluid depth between 350 and $450 \mathrm{~m}$. Tests are performed on an $f$ plane $\left(f=1.0 \times 10^{-4} \mathrm{~s}^{-1}\right)$ for the inviscid equations $(\nu=0)$, with a domain size of $12000 \mathrm{~km} \times$ $12000 \mathrm{~km}$. We use a time step of $25 \mathrm{~s}$.

Figure 14 shows the zonal velocity field on the different grids, and its evolution in time. It is visible that the eddy size increases with increasing time, and that the fields are finer resolved but qualitatively equivalent on the refined grids.

We evaluate the energy spectra to get a better impression of the influence of grid refinement to the turbulent cascade. To obtain an approximation of the kinetic energy spectra on the refined grids, we evaluate only the grid points in the initial, unrefined grid, like we already did in section $3 b$. Since it is difficult to read information from the two dimensional energy spectra, we present the spectra in one-dimensional form. To this end, we take the average of all spectral coefficients that represent a wavelength $\lambda=\sqrt{\lambda_{x}^{2}+\lambda_{y}^{2}}$ within an interval between $L_{x} / i$ and $L_{x} /(i+1), \forall i=1, \ldots, 59$.

Figure 15 shows the one-dimensional kinetic energy spectra of the model runs calculated for the full domain and for the left- and the right-hand side of the domain. The spectra of the partly refined grids calculated over the full domain lie between the $\mathrm{H}_{0}$ and $\mathrm{H}_{1}$ spectra and are therefore closer to the expected cubic decay rate compared to the coarse uniform simulation. The grid that was refined in two refinement levels has a different higher energy spectra for the very small wavelength; a behavior we would expect for a finer resolved model simulation. While the spectra on refined grids are slightly increased for the coarse left-hand side, they are slightly decreased for the fine right-hand side of the grid compared to uniform simulations at the same resolution if calculated only in the specific hand side of the grid. All spectra look reasonable and no spurious accumulation of energy is visible for the refined grids.

\section{d. Topography and grid refinement}

We analyze simulations of a zonal geostrophic flow over an isolated mountain on refined grids. The test is known from Williamson et al. (1992) (test case 5) and consists of a global steady-state nonlinear zonal geostrophic flow that is perturbed by a mountain introduced into the Northern Hemisphere, behind which Rossby waves form and propagate over the globe.

The steady case specifies a zonal westerly flow with a maximum velocity of $\sim 20 \mathrm{~m} \mathrm{~s}^{-1}$ and a fluid depth varying between 5960 and $7960 \mathrm{~m}$. The test is performed without viscosity $(\nu=0)$. 

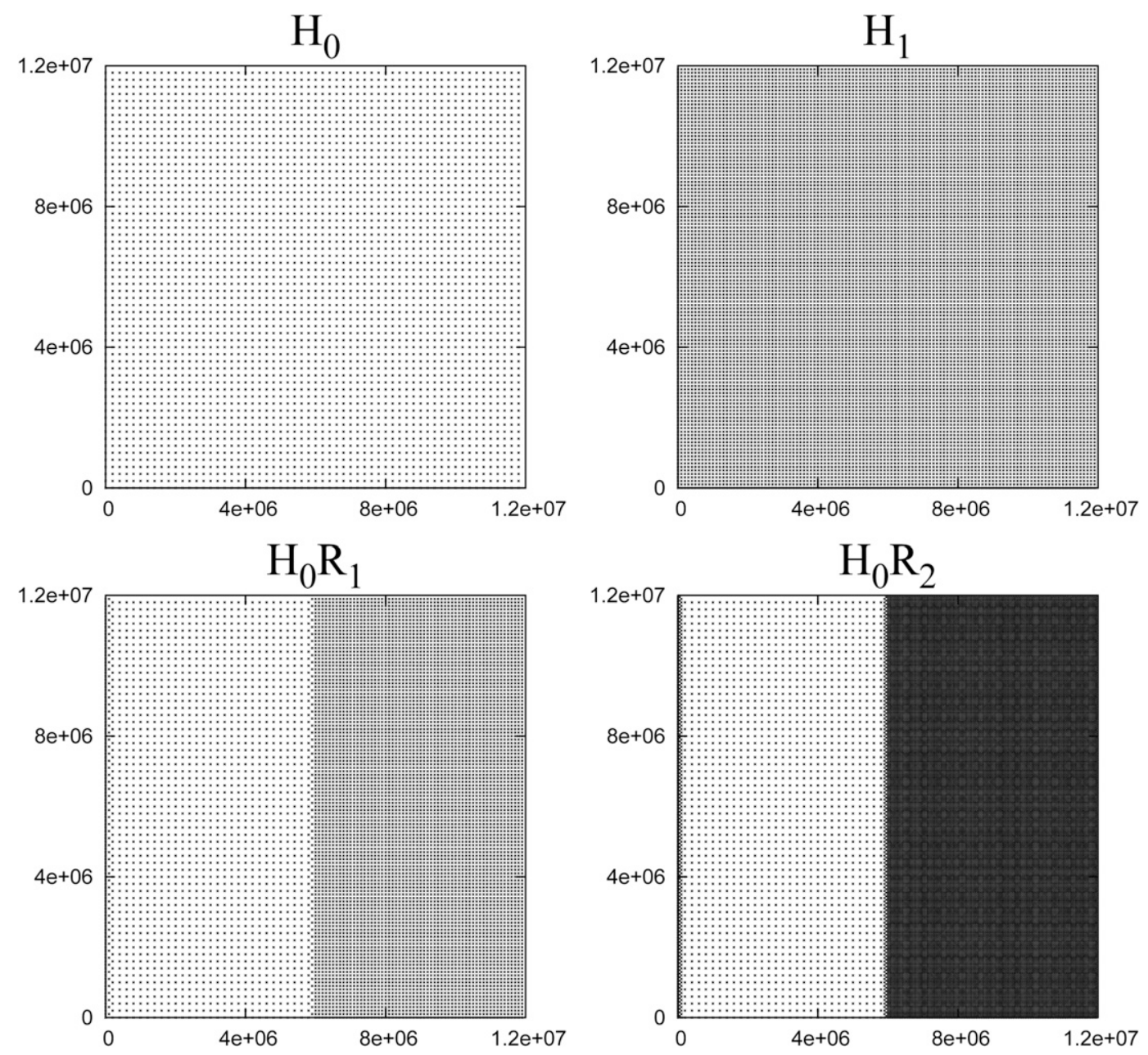

FIG. 13. Turbulent decay affected by grid refinement test: positions of the vertices in the grids.

We perform simulations on seven different grids. Three of them are uniform icosahedral geodesic grids $\left(\mathrm{H}_{0}, \mathrm{H}_{1}\right.$, and $\left.\mathrm{H}_{2}\right)$. Additionally, we use four refined grids in which the resolution around the mountain is increased by a factor of $2\left(\mathrm{H}_{0} \mathrm{Re}_{1}, \mathrm{H}_{1} \mathrm{Re}_{1}\right)$ or $4\left(\mathrm{H}_{0} \mathrm{Re}_{2}, \mathrm{H}_{1} \mathrm{Re}_{2}\right)$ starting from the $\mathrm{H}_{0}$ or the $\mathrm{H}_{1}$ grid. The refinement area of the $\mathrm{H}_{0} \mathrm{Re}_{1}$ and the $\mathrm{H}_{1} \mathrm{Re}_{1}$ grid spans the rectangular between the longitude values of $125^{\circ}-55^{\circ} \mathrm{W}$ and the latitude values of $10^{\circ}-55^{\circ} \mathrm{N}$. The refinement area of the first refinement level of the $\mathrm{H}_{0} \mathrm{Re}_{2}$ and the $\mathrm{H}_{1} \mathrm{Re}_{2}$ grid spans the rectangular between $130^{\circ} \mathrm{W}-30^{\circ} \mathrm{E}$ and $25^{\circ} \mathrm{S}-$ $70^{\circ} \mathrm{N}$. The second refinement level spans between $125^{\circ}-$ $55^{\circ} \mathrm{W}$ and $10^{\circ}-55^{\circ} \mathrm{N}$. Some of the grids are plotted in Fig. 16. Details on the grids are given in Table 1. We use a time step of $25 \mathrm{~s}$.

Figure 17 shows the difference in height of the simulations at day 15 , compared to a reference simulation on a uniform icosahedral grid of high resolution with an averaged edge length of $120 \mathrm{~km}$. Inside of the refined areas the error is reduced significantly and spurious behavior is not visible at the transition zones between different refinement levels, but outside of the refined areas improvements are small.

Figure 18 shows the kinetic energy and enstrophy spectra calculated at day 15 and day 100 for the different grids. The procedure to calculate the kinetic energy spectra on a sphere is described in the appendix. At day 15, all spectra are still in good agreement. At day 100, the refined grids produce a spectra closer to the runs on uniform grids with higher resolution and closer to the theoretically expected decay rate, compared to their unrefined partners, not only for the smallest wavenumbers. The turbulent decay seems to be improved on the long term.

\section{e. Lateral boundaries and grid refinement}

We perform classical numerical ocean tests consisting of wind-driven Munk gyres in an idealized planar setup 

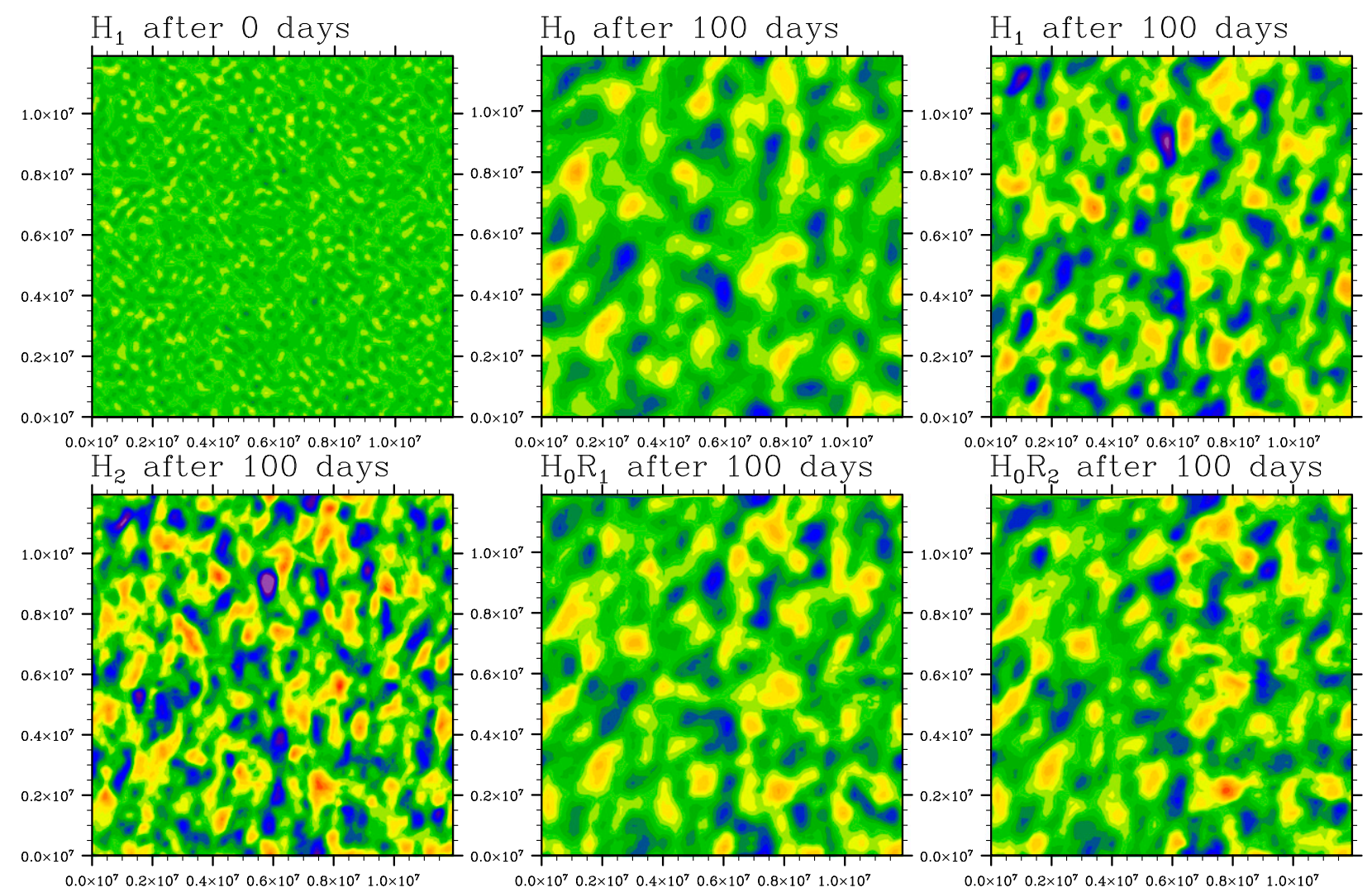

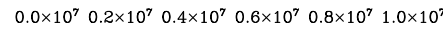

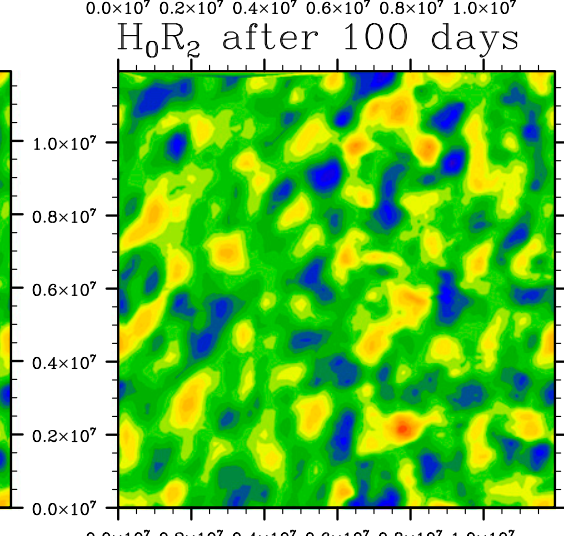

$0.0 \times 10^{7} \quad 0.2 \times 10^{7} \quad 0.4 \times 10^{7} \quad 0.6 \times 10^{7} \quad 0.8 \times 10^{7} 1.0 \times 10^{7}$

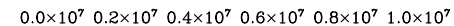

$0.0 \times 10^{7} \quad 0.2 \times 10^{7} \quad 0.4 \times 10^{7} \quad 0.6 \times 10^{7} \quad 0.8 \times 10^{7} 1.0 \times 10^{7}$

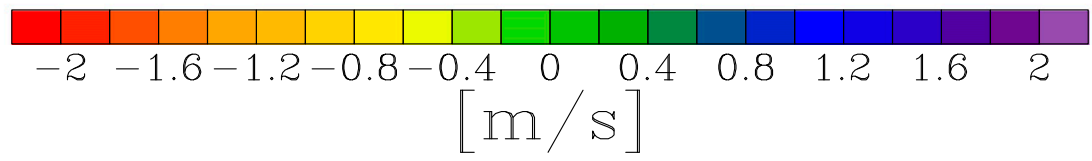

FIG. 14. Turbulent decay affected by grid refinement: zonal velocity of the initial conditions on the (top left) $\mathrm{H}_{1}$ grid, and after 100 days on the (from top left to bottom right) $\mathrm{H}_{0}, \mathrm{H}_{1}, \mathrm{H}_{2}, \mathrm{H}_{0} \mathrm{R}_{1}$, and $\mathrm{H}_{0} \mathrm{R}_{2}$ grids. It is visible that the eddy size increases with increasing time, and that the fields are finer resolved but qualitatively equivalent on the refined grids. No spurious behavior is visible at the transition zones.

and in a North Atlantic domain with real world topography and coast lines. A wind forcing induces a clockwise circulation. Because of the change of the Coriolis parameter in the meridional direction the gyre is intensified toward the western boundary, and a western boundary current develops (Pedlosky 1996). The tests are realized with free-slip boundary conditions $(\mathbf{u} \cdot \mathbf{n}=$ 0 , and $\partial \mathbf{u} / \partial \mathbf{n}=0$ on the boundary $\partial \Omega$ ). We are particularly interested in the impact of grid refinement on the representation of western boundary currents. Piggott et al. (2009) could already show the huge potential to reduce the number of nodes when using adaptive grid refinement for a standard Galerkin finite-element discretization with continuous piecewise-linear basis functions.

The planar domain has the dimensions of $1000 \mathrm{~km} \times$ $1000 \mathrm{~km}$ and is located in the Northern Hemisphere. The test case is initialized with a constant water depth of
$1000 \mathrm{~m}$ and zero velocity. The Coriolis parameter is given in $\beta$-plane approximation by

$$
f=10^{-4} \mathrm{~s}^{-1}+4.0 \times 10^{-11} \mathrm{ys}^{-1},
$$

where $y$ is the meridional coordinate. Wind forcing is introduced to the differential equation for velocity through the following forcing term:

$$
\tau=\frac{\tau^{s}}{H}
$$

where $\tau^{s}$ is the surface wind forcing and $H$ is the fluid depth.

While the meridional wind forcing is zero, the zonal wind forcing is set to

$$
\tau_{x}^{s}=-\tau_{0} \times 10^{-3} \times \cos \left(\frac{\pi y}{L}\right),
$$



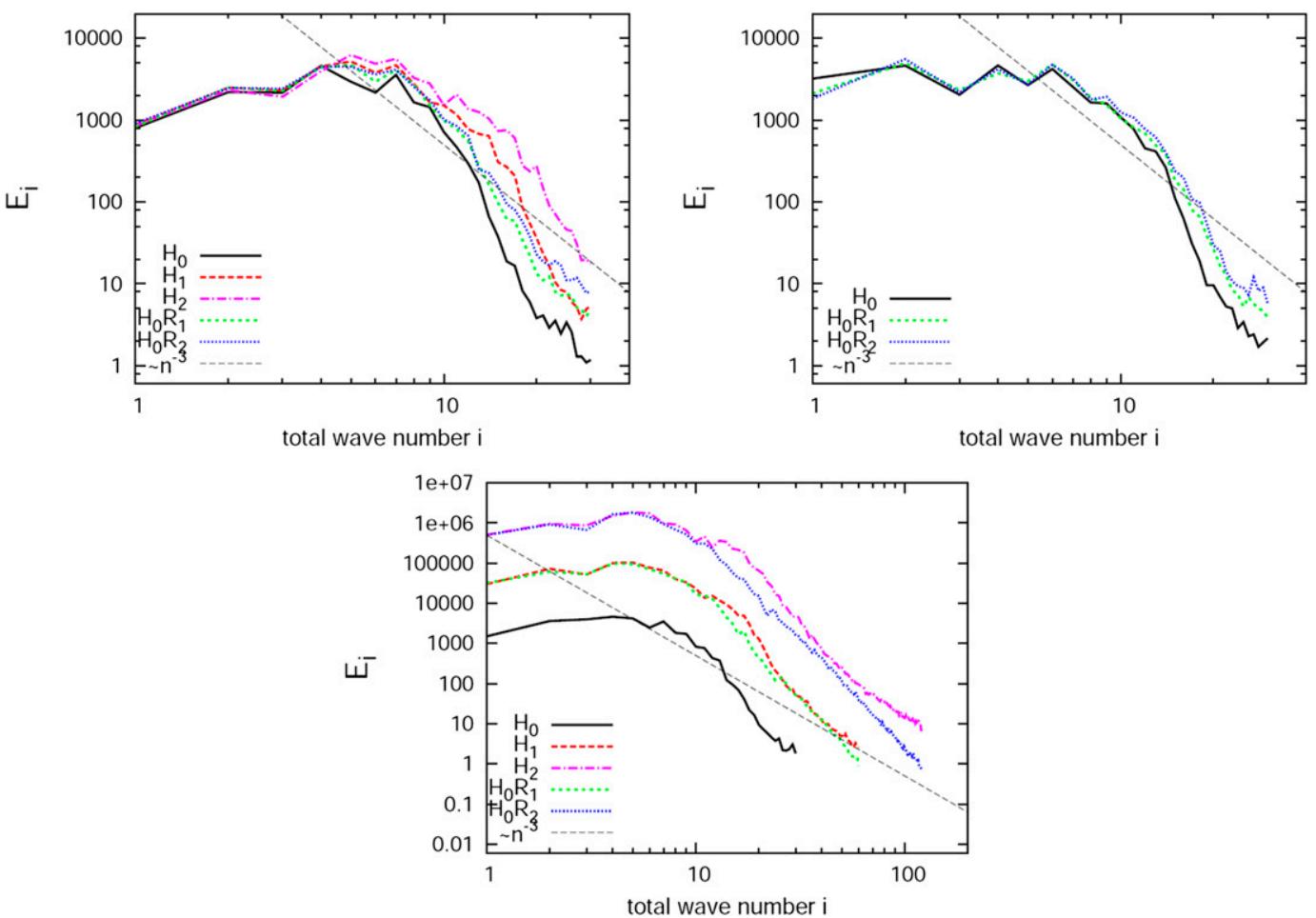

FIG. 15. Turbulent decay affected by grid refinement: one-dimensional kinetic energy spectra after 100 days for the full domain, and (from left to right) for the left- and the right-hand side of the domain. The spectra for the full domain were calculated from a discrete Fourier transformation on the original grid with $60 \times 60$ vertices for all grids, to allow a direct comparison of the spectra. The spectra of the partly refined grids lie between the $\mathrm{H}_{0}$ and $\mathrm{H}_{1}$ grid and are therefore closer to the expected cubic decay rate compared to the coarse uniform simulation. The spectra for the leftand the right-hand side of the domain were calculated from a discrete Fourier transformation on a grid with the respective local resolution. While the spectra on refined grids are slightly increased for the coarse left half, they are slightly decreased for the fine right-hand side of the grid compared to uniform simulations at the same resolution.

where $L$ is the length of the domain in meridional direction. We use a time step of $10 \mathrm{~s}$.

We use three different grids for the simulations (Figure 19). One grid in which the area that is important for the representation of the western boundary current is refined and two grids that offers a coarse and fine uniform resolution. The refined and the fine grid have the same maximal resolutions.

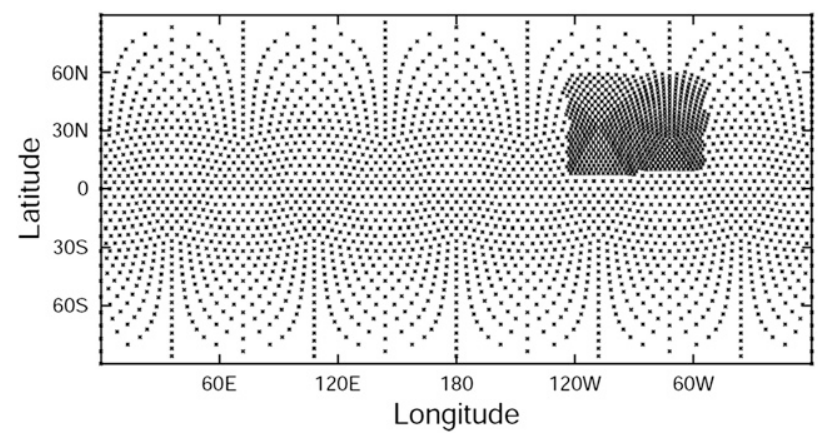

While energy is introduced to the system via the wind forcing, the energy is reduced by diffusion. The value used for the eddy viscosity $\nu$ and the wind forcing $\tau_{0}$ need to be balanced at a realistic level for the resulting velocity field. In principle, we want to use as little viscosity as possible, since viscosity dampens the flow patterns and reduces details. On the other hand, the width of the Munk layer at the western boundary is fixed by the value of eddy

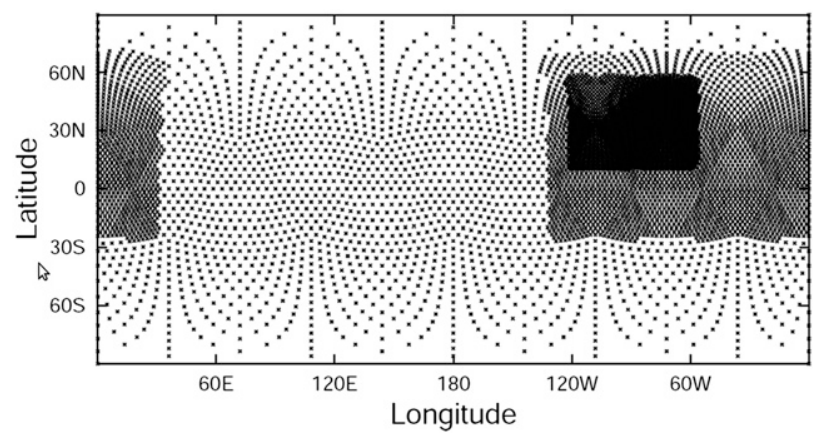

FIG. 16. Topography and grid refinement: positions of the vertices in the (left) $H_{1} R_{1}$ and (right) $H_{1} \operatorname{Re}_{2}$ grid. 
TABLE 1. Properties of the grids used for the topography and grid refinement test.

\begin{tabular}{lcc}
\hline \hline & No. of cells & $\begin{array}{c}\text { Typical edge length } \\
\text { on the sphere }(\mathrm{km})\end{array}$ \\
\hline $\mathrm{H}_{0}$ & 1280 & 960 \\
$\mathrm{H}_{0} \mathrm{Re}_{1}$ & 1596 & $480-960$ \\
$\mathrm{H}_{0} \mathrm{Re}_{2}$ & 3636 & $240-960$ \\
$\mathrm{H}_{1}$ & 5120 & 480 \\
$\mathrm{H}_{1} \mathrm{Re}_{1}$ & 6182 & $240-480$ \\
$\mathrm{H}_{1} \mathrm{Re}_{2}$ & 13792 & $120-480$ \\
$\mathrm{H}_{2}$ & 20480 & 240 \\
\hline
\end{tabular}

viscosity, and the Munk layer needs to be resolved by at least two grid cells. This leads to a condition for the minimal viscosity that we are allowed to use in our simulations, which is dependent on the grid resolution at the western boundary. The constraint that assures that the
Munk layer is resolved by at least $N$ grid points in a global finite-difference model is given by

$$
\nu>3.82 \times 10^{-12}(N \Delta s)^{3} \cos (\theta) \mathrm{m}^{-1} \mathrm{~s}^{-1},
$$

where $\Delta s$ is the lattice spacing and $\theta$ is the latitude (Griffies 2004). We calculate the minimal values needed for eddy viscosity, to resolve the Munk layer with two grid cells, with an equation similar to Eq. (3) derived and tested for the $\beta$-plane approximation and free-slip boundaries. Using a lower viscosity leads to an insufficiently resolved western boundary current. We use $\nu=100.0 \mathrm{~m}^{2} \mathrm{~s}^{-1}$ and $\tau_{0}=0.1 \mathrm{~m}^{2} \mathrm{~s}^{-1}$. The number of cells is 512 in the coarse, 730 in the refined, and 2048 in the fine grid.

Figure 20 shows the equilibrated height field of the model runs on the three grids after one year. It is visible that the western boundary is not properly resolved in the
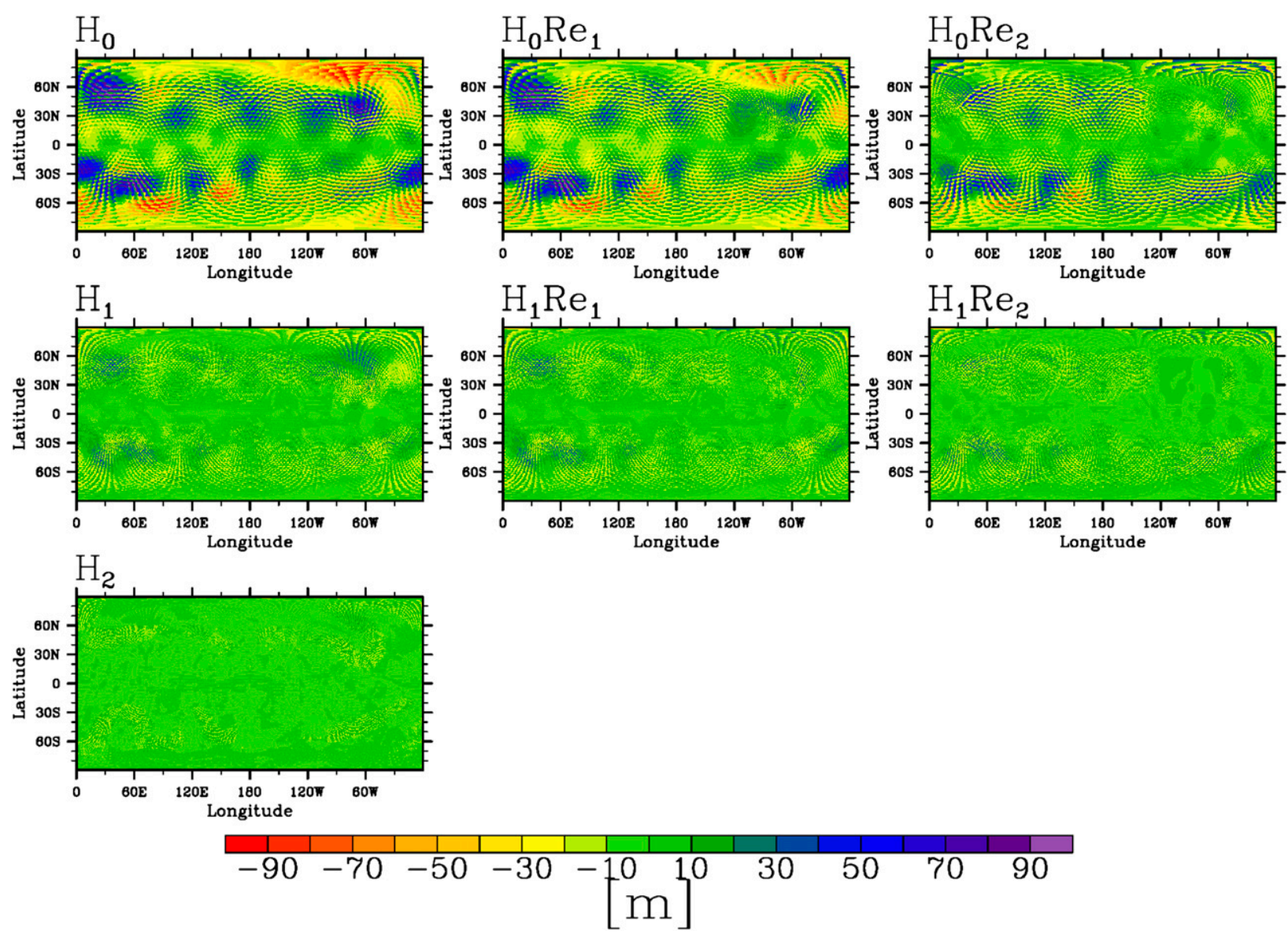

FIG. 17. Topography and grid refinement: error of height on the different grids [(from top left to bottom right) $\mathrm{H}_{0}, \mathrm{H}_{0} \mathrm{Re}_{1}, \mathrm{H}_{0} \mathrm{Re}_{2}, \mathrm{H}_{1}$, $\mathrm{H}_{1} \mathrm{Re}_{1}, \mathrm{H}_{1} \mathrm{Re}_{2}$, and $\mathrm{H}_{2}$ ] after 15 days compared to a reference run with higher resolution. Inside of the refined areas the error is reduced significantly and spurious behavior is not visible at the transition zones between different refinement levels, but outside of the refined areas improvements are small. Small-scale noise pattern are caused by the mapping, which is necessary to compare the results on different grids, and not so much by numerical noise within the simulations. 

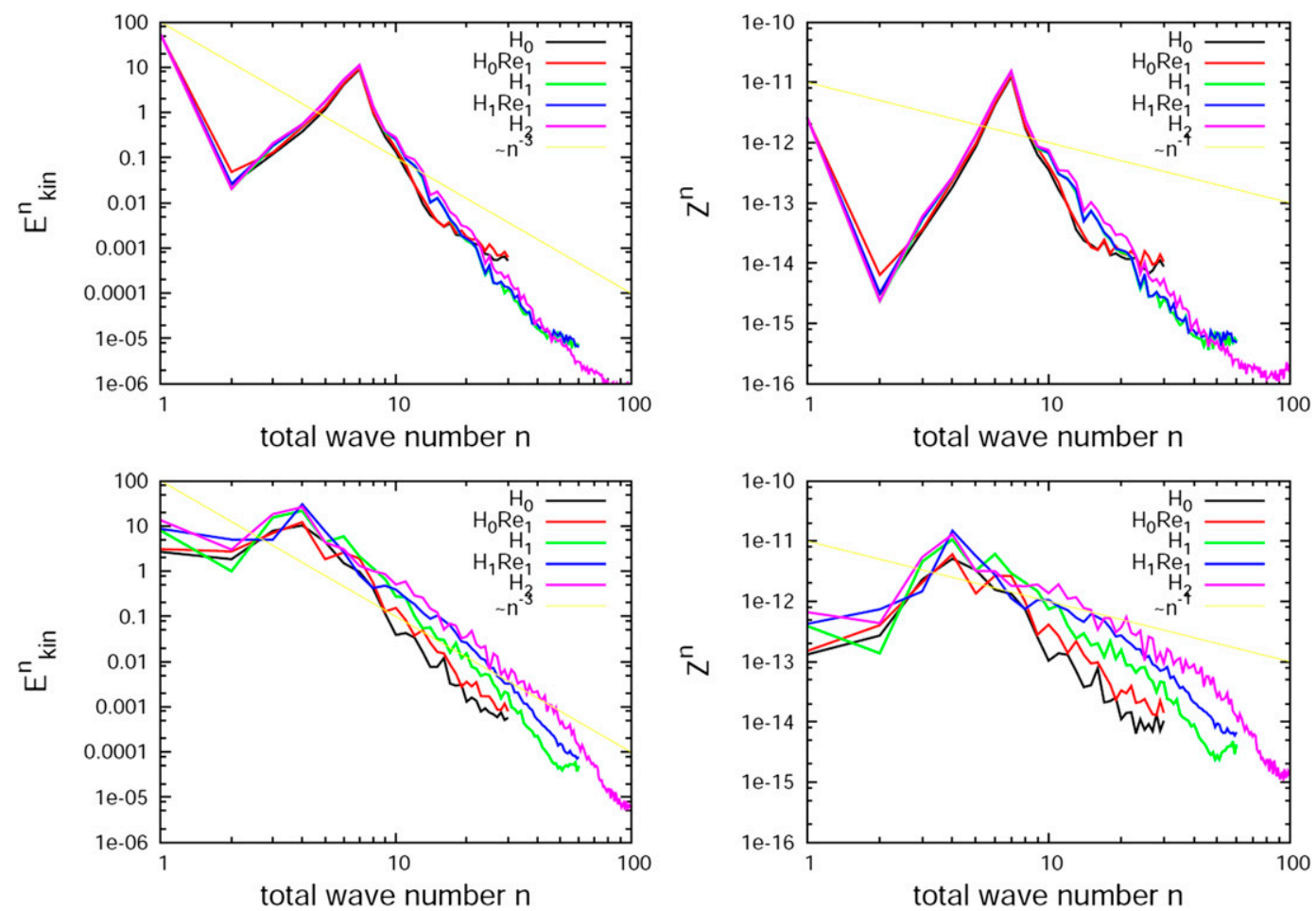

FIG. 18. Topography and grid refinement: (left) one-dimensional energy and (right) enstrophy spectra on the sphere after (top) 15 and (bottom) 100 days for different grids. At day 100, the refined grids produce a spectra closer to the runs on uniform grids with higher resolution and closer to the theoretically expected decay rate, compared to their unrefined partners, not only for the smallest wavenumbers.

coarse simulation. In contrast, the height field of the refined grid is represented smoothly along the western boundary. In comparison to the uniform, fine simulation, the height pattern of the refined simulation shows a clear improvement compared to the height field of the uniform, coarse simulation. These results are consistent with the results in Piggott et al. (2009) for adaptive mesh refinement.
Next we simulate an ocean basin shaped like the Atlantic Ocean, but with a domain that is cut at the equator and at $58^{\circ} \mathrm{N}$. The real-world topography is cut at $1000-\mathrm{m}$ depth. An artificial wind forcing that is balanced by bottom friction induces a steady circulation. We use two different grids. A uniform grid with a typical edge length of $120 \mathrm{~km}$, and the same grid refined at the western boundary, with a typical edge length of $60 \mathrm{~km}$ in the fine
Coarse

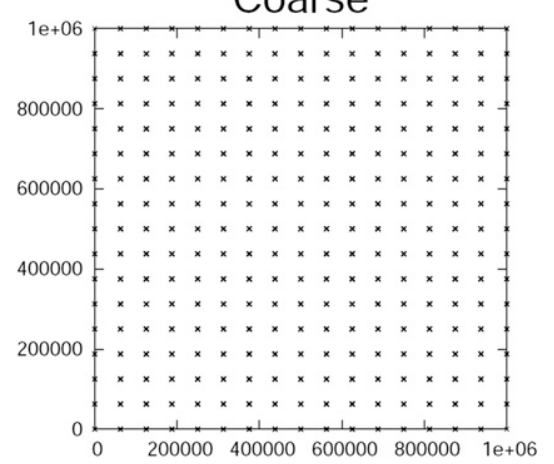

Refined

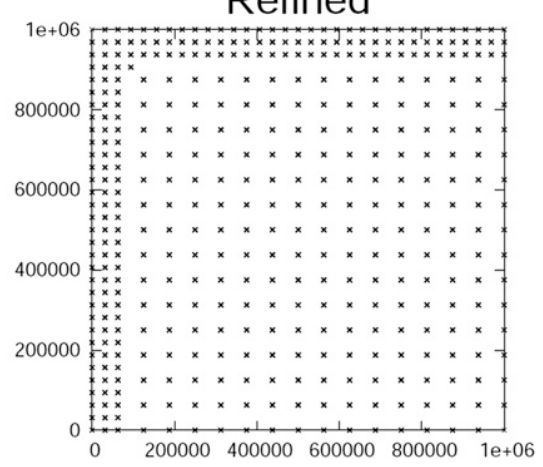

Fine

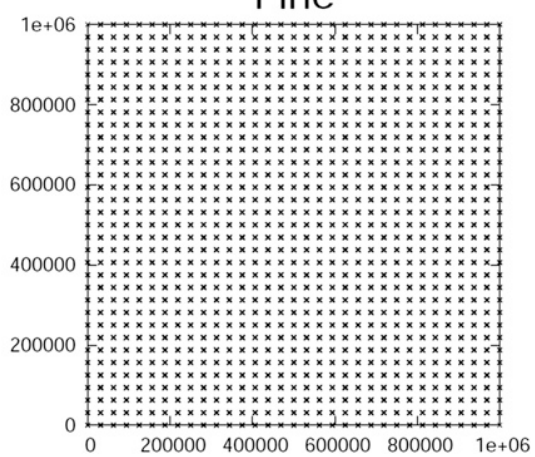

FIG. 19. Lateral boundaries and grid refinement: positions of the vertices in the coarse, the refined, and the fine grids used for the planar Munk gyre test. 


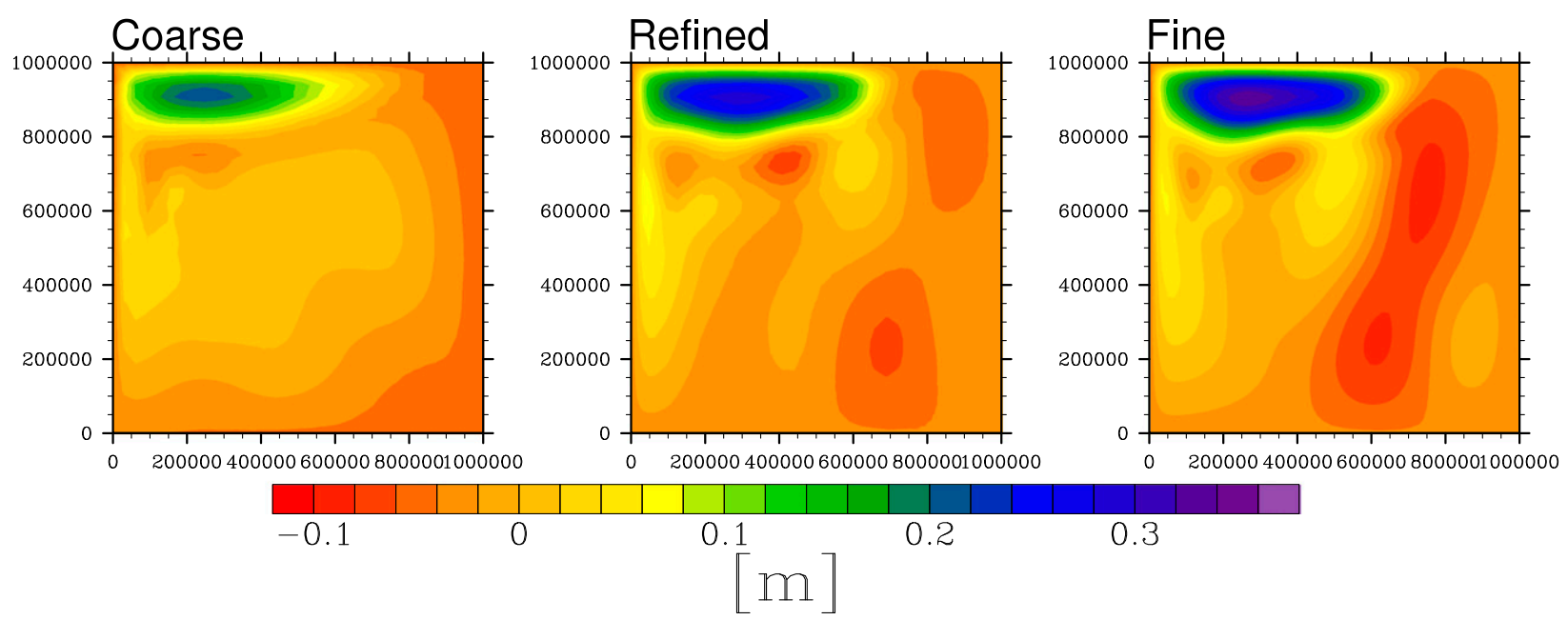

FIG. 20. Lateral boundaries and grid refinement: (from left to right) equilibrium surface elevation of the planar Munk gyre simulations on the coarse, the refined, and the fine grids. Grid refinement along the boundary leads to a clear improvement compared to the simulation with uniform, coarse resolution.

part of the grid (plotted in Fig. 21). We use a time step of $20 \mathrm{~s}$.

Simulations are initialized with zero surface elevation and zero velocity. The zonal wind forcing is given by

$$
\tau_{\lambda}^{s}= \begin{cases}-\tau_{0} \times 10^{-3} \times \cos (4 \theta) & \text { if } \quad \theta<45^{\circ} \\ 0 & \text { if } \quad \theta \geq 45^{\circ}\end{cases}
$$

where the meridional wind forcing is zero.

Figure 22 shows the equilibrated steady surface elevation for model simulations with $\tau_{0}=1.0 \mathrm{~m}^{2} \mathrm{~s}^{-1}$ and $\nu=6655.0 \mathrm{~m}^{2} \mathrm{~s}^{-1}$ for the refined and $\tau_{0}=2.0 \mathrm{~m}^{2} \mathrm{~s}^{-1}$ and $\nu=53240.0 \mathrm{~m}^{2} \mathrm{~s}^{-1}$ for the unrefined grids. The increase of the local resolution at the western boundary allows the use of a lower eddy viscosity. If we use the same eddy viscosity on the uniform grid, that we use for the refined simulation $\left(\nu=6655.0 \mathrm{~m}^{2} \mathrm{~s}^{-1}\right)$, the Munk layer is not properly resolved. Since we use a lower viscosity we dissipate less energy and we can use a weaker and therefore more realistic wind forcing to obtain very similar values for the velocity. We argue that the height field in the refined model run shows a smaller Munk layer, with more details compared to the uniform run, and is therefore preferable. Grid refinement leads to only a small increase in computational costs, since the increase of the number of degrees of freedom is fairly small (cf. 7250 grid cells in the refined vs 5783 cells in the uniform simulation).

From the Munk gyre test one can learn that refining the grid and adapting the physics, here the change in the viscosity coefficient, has to go hand in hand in order to obtain an improved model solution. Here it was exceptionally simple because we just had to satisfy the lower bound for viscosity [Eq. (3)], but for the general case such kind of information is not available.

\section{Conclusions}

Our results provide numerical evidence only and the conclusions are limited to the $P_{1}^{\mathrm{DG}} P_{2}$ finite element.

The simulations of a zonal geostrophic flow (section 3a) suggest that grids of variable resolution do not perturb the representation of the geostrophic balance, but they can increase the zonal asymmetry which will induce errors. For the specific test case, the convergence properties are changed as well.

As expected, the simulations of wave packets (section $3 b)$ show that linear gravity waves with wavelengths less than 5 times the coarser grid spacing are strongly reflected and scattered at the transition between coarse and fine grids, while waves that are well resolved on the

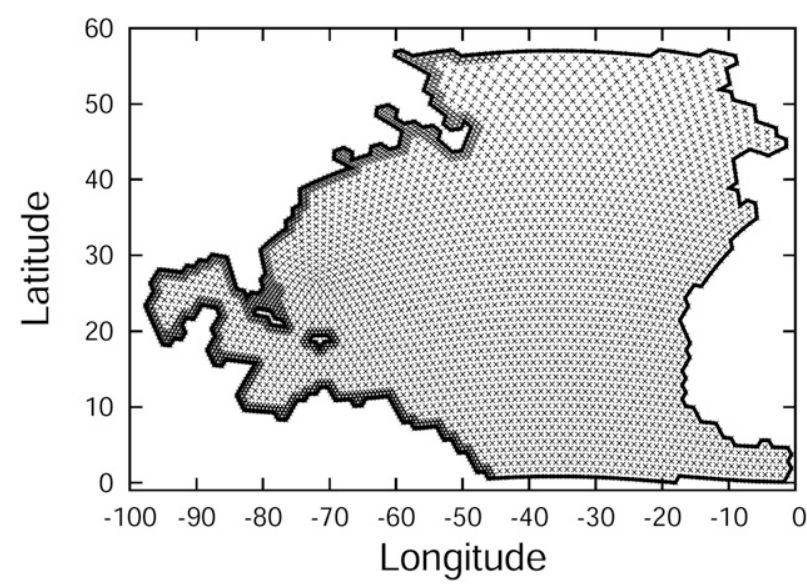

FIG. 21. Lateral boundaries and grid refinement: vertices of the refined grid used for the Atlantic shaped Munk gyre. The black line marks the coastline. 

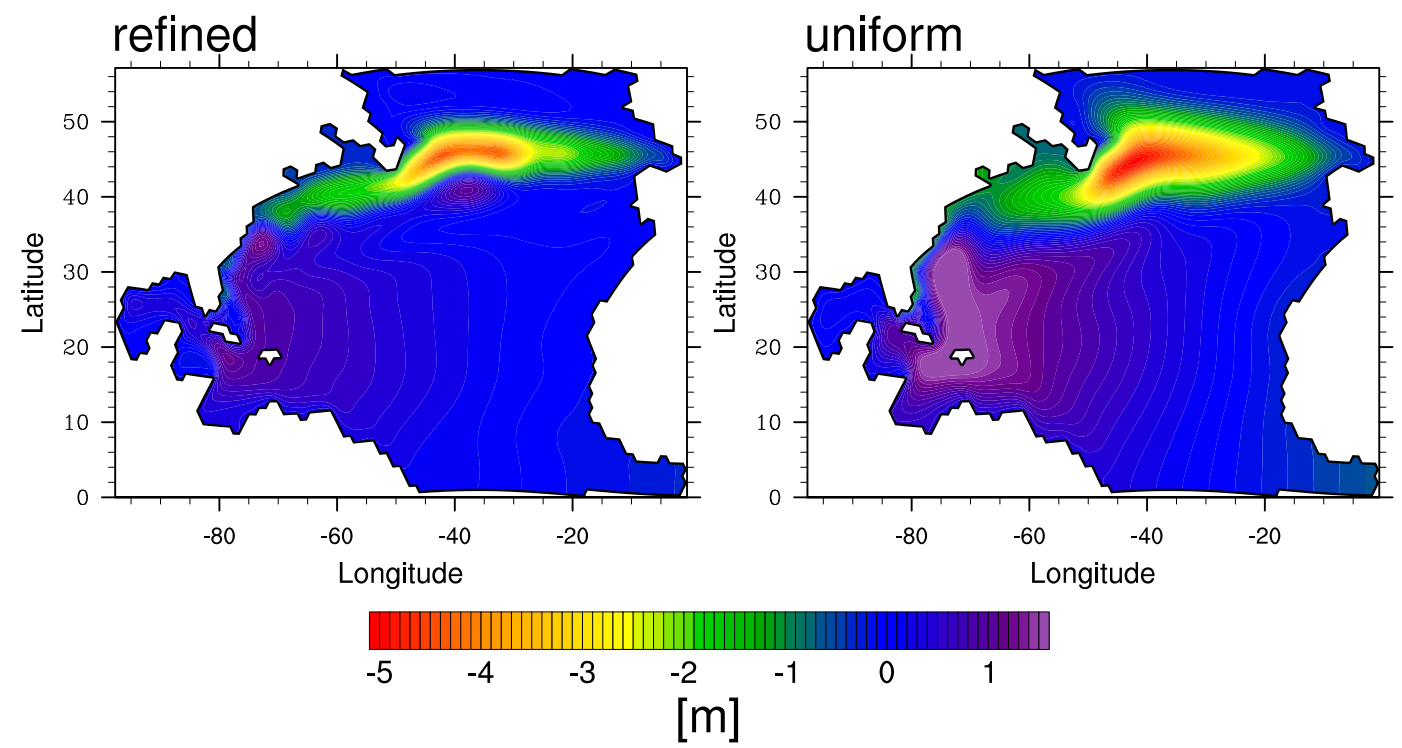

FIG. 22. Lateral boundaries and grid refinement: equilibrium surface elevation in the Atlantic-shaped Munk gyre after one year of simulations (left) with and (right) without refined western boundary. We used $\nu=6655.0 \mathrm{~m}^{2} \mathrm{~s}^{-1}$ in the refined, and $\nu=53240.0 \mathrm{~m}^{2} \mathrm{~s}^{-1}$ in the unrefined model simulations. Because of the lower viscosity, the refined model run shows a smaller Munk layer and more details compared to the uniform run.

coarse grid are hardly effected. The errors are fairly independent of the structure of the transition zone and the change of resolution between fine and coarse parts of the grids. Even if changes might appear to be small when looking at the physical fields only, errors could be identified by looking at the spectra (Figs. 8 and 9). Therefore, it is likely that many errors in the wave propagation will be invisible but still apparent when using grids of variable resolution. Nevertheless, the simulations of randomly initialized physical fields (section $3 \mathrm{c}$ but also section $3 \mathrm{~d}$ ) show that grid refinement does not have a strong influence on the turbulent cascades in the considered finite-element setup. While the energy spectra of the full grid appears to be improved, small changes are visible for the local spectra within the fine and the coarse region with a slight decrease and increase of kinetic energy, respectively. The error in energy conservation is influenced by grid refinement, but it is typically getting smaller compared to the error on the corresponding unrefined grids (sections $3 a$ and $3 b)$.

The simulations of flow over topography (section 3d) can be seen as an example that it is not necessarily true that the global error is reduced when grid refinement is able to reduce the local error around an important local feature, although the local feature is strongly influencing the whole simulation. These results are consistent with the results in Ringler et al. (2011) where multiresolution simulations with a smooth transition zone between the coarse and the fine part of the grid are performed with a model based on a finite-volume scheme. Ringler et al. showed that the global error of simulations is mostly dependent on the resolution of the coarse part of the grid, when simulating the same test case.

For the representation of boundary currents a careful use of grid refinement allows a fair improvement of the solution while the increase in computational cost appears to be comparably small. The tests approve that grid refinement will only reveal its full potential if it goes along with a simultaneous change of the parameterization schemes, here a reduction of the kinematic viscosity that was possible due to an increased resolution within the Munk layer.

In summary, grid refinement appears to be promising for this particular setup and we did not discover profound problems when using variable resolution, even if abrupt changes in grid resolution are used. But it also became clear that grid refinement is not a panacea and that many further studies will be needed to unleash its full potential and minimize errors. On a broader perspective our results advocate a deeper investigation of static local grid refinement as a viable alternative to timevarying adaptive grid refinements or at least as an intermediate step toward adaptive grids. This holds in particular for applications where regions of interest can be identified a priori, as it is the case in ocean modeling. Many open questions remain, for example how to compare different (static) refinement procedures such as $h$ 
refinement, $p$ refinement, $r$ refinement, and nesting. ${ }^{1}$ It is not even clear how to carry out a sensible comparison between these different refinement strategies. The metrics for this sort of model intercomparison constitute a challenge in itself.

Acknowledgments. We thank Juan Pedro Mellado and the anonymous reviewers for very useful feedback on previous versions of this paper and Todd Ringler for interesting comments and discussions.

\section{APPENDIX}

\section{Diagnostics for Energy and Enstrophy}

\section{a. Energy conservation}

We calculate the global energy at time step $j$ using the following formula:

$$
E^{j}=\int_{\Omega}\left[\frac{1}{2} h^{j} \mathbf{u}^{j} \cdot \mathbf{u}^{j}+\frac{1}{2} g\left(h^{j} h^{j}-h_{b}^{j} h_{b}^{j}\right)\right] d x .
$$

We compute the relative error for total energy at time step $j$ using the initial value as a reference:

$$
\eta_{E}\left(t_{j}\right)=\frac{\left|E^{0}-E^{j}\right|}{E^{0}}
$$

\section{b. Kinetic energy and enstrophy spectra on plane and sphere}

The model runs are evaluated with kinetic energy and enstrophy spectra. Using a discrete Fourier transformation, we calculate the spectral coefficients $\hat{\phi}_{i j}$ of a twodimensional physical field $\phi$ on regular equidistant grids on the plane via

$$
\hat{\phi}_{k l}=\sum_{m=0}^{N_{x}-1} \sum_{p=0}^{N_{y}-1} \phi_{m p} \exp \left(-2 \pi i \frac{m k}{N_{x}}\right) \exp \left(-2 \pi i \frac{p l}{N_{y}}\right),
$$

where $\phi_{m p}=\phi\left(x_{m}, y_{p}\right)$ is the value of the physical field at a given grid point $\left(\forall m=1,2,3, \ldots, N_{x}\right.$, and $\forall p=1,2,3$, $\left.\ldots, N_{y}\right)$. We define the factor $1 / N_{x} N_{y}$ to be introduced in the inverse transformation back to physical space.

By calculating the spectral coefficients for the two components of the velocity fields $\left(\hat{u}_{k l}\right.$ and $\left.\hat{v}_{k l}\right)$, we obtain the two-dimensional spectra of the kinetic energy:

\footnotetext{
${ }^{1}$ The $h$ refinement: insertion of new grid cells, as considered here; $p$ refinement: changing of the local order of the numerical model; $r$ refinement: modification of the positions of the grid cells; and nesting: local insertion of a whole model at higher resolution.
}

$$
E_{k l}=\frac{1}{4}\left(\left|\hat{u}_{k l}\right|^{2}+\left|\hat{v}_{k l}\right|^{2}\right)
$$

To calculate the spectra of the kinetic energy and enstrophy on the sphere, we proceed as follows. In a first step, the relative vorticity and divergence are calculated as curl and divergence of the velocity. In a second step, vorticity and divergence are mapped onto a Gaussian grid. In a third step, the spectral coefficients of vorticity and divergence $\left(\zeta_{n}^{m}\right.$ and $\left.\delta_{n}^{m}\right)$ are calculated. Steps 2 and 3 are performed using the Climate Data Operator tools (CDO; Müller and Schulzweida 2010).

The spectra of kinetic energy $E_{\text {kin }}^{n}$ and enstrophy $Z^{n}$ are given by

$$
\begin{aligned}
E_{\mathrm{kin}}^{n}= & \frac{a_{e}^{2}}{4 n(n+1)}\left(\left|\zeta_{n}^{0}\right|^{2}+\left|\delta_{n}^{0}\right|^{2}+2 \sum_{m=1}^{n}\left|\zeta_{n}^{m}\right|^{2}\right. \\
& \left.+2 \sum_{m=1}^{n}\left|\delta_{n}^{m}\right|^{2}\right), \\
Z^{n}= & \frac{1}{4}\left(\left|\zeta_{n}^{0}\right|^{2}+\sum_{m=1}^{n} 2\left|\zeta_{n}^{m}\right|^{2}\right),
\end{aligned}
$$

where $n$ is the wavenumber. A derivation of Eq. (A2) is given in Jakob et al. (1993).

\section{REFERENCES}

Baumgardner, J. R., and P. O. Frederickson, 1985: Icosahedral discretization of the two-sphere. SIAM J. Numer. Anal., 22, 1107-1115, doi:10.1137/0722066.

Behrens, J., 2006: Adaptive Atmospheric Modeling: Key Techniques in Grid Generation, Data Structures, and Numerical Operations with Applications. Springer, $214 \mathrm{pp}$.

Bonaventura, L., and T. Ringler, 2005: Analysis of discrete shallowwater models on geodesic Delaunay grids with C-type staggering. Mon. Wea. Rev., 133, 2351-2373, doi:10.1175/MWR2986.1.

Comblen, R., J. Lambrechts, J.-F. Remacle, and V. Legat, 2010: Practical evaluation of five partly discontinuous finite element pairs for the non-conservative shallow water equations. Int. J. Numer. Methods Fluids, 63, 701-724, doi: 10.1002/fld.2094.

Cotter, C. J., and D. A. Ham, 2011: Numerical wave propagation for the triangular P1DG-P2 finite element pair. J. Comput. Phys., 230, 2806-2820, doi:10.1016/j.jcp.2010.12.024.

$\longrightarrow,-$, and C. C. Pain, 2009a: A mixed discontinuous/continuous finite element pair for shallow-water ocean modelling. Ocean Modell., 26, 86-90, doi:10.1016/j.ocemod.2008.09.002.

,,--- , and S. Reich, 2009b: LBB stability of a mixed Galerkin finite element pair for fluid flow simulations. J. Comput. Phys., 228, 336-348, doi:10.1016/j.jcp.2008.09.014.

Danilov, S., G. Kivman, and J. Schröter, 2004: A finite-element ocean model: Principles and evaluation. Ocean Modell., 6, 125-150, doi:10.1016/S1463-5003(02)00063-X.

Düben, P. D., P. Korn, and V. Aizinger, 2012: A discontinuous/ continuous low order finite element shallow water model on the sphere. J. Comput. Phys., 231, 2396-2413, doi:10.1016/ j.jcp.2011.11.018. 
Griffies, S. M., 2004: Fundamentals of Ocean Climate Models. Princeton University Press, 496 pp.

Harris, L. M., and D. R. Durran, 2010: An idealized comparison of one-way and two-way grid nesting. Mon. Wea. Rev., 138, 2174 2187, doi:10.1175/2010MWR3080.1.

Jakob, R., J. J. Hack, and D. L. Williamson, 1993: Solutions to the shallow water test set using the spectral transform method. NCAR Tech. Note TN-388+STR, NCAR, 83 pp.

Le Roux, D. Y., A. Staniforth, and C. A. Lin, 1998: Finite elements for shallow-water equation ocean models. Mon. Wea. Rev., 126, 1931-1951, doi:10.1175/1520-0493(1998)126<1931: FEFSWE $>2.0 . \mathrm{CO} ; 2$.

Long, D., and J. Thuburn, 2011: Numerical wave propagation on non-uniform one-dimensional staggered grids. J. Comput. Phys., 230, 2643-2659, doi:10.1016/j.jcp.2010.12.040.

Maddison, J., C. Cotter, and P. Farrell, 2011: Geostrophic balance preserving interpolation in mesh adaptive linearised shallowwater ocean modelling. Ocean Modell., 37, 35-48, doi:10.1016/ j.ocemod.2010.12.007.

McWilliams, J. C., 2006: Fundamentals of Geophysical Fluid Dynamics. Cambridge University Press, 266 pp.

Müller, R., and U. Schulzweida, 2010: Climate data operators, version 1.4.5.1. NOAA.

Pedlosky, J., 1996: Ocean Circulation Theory. Springer-Verlag, 453 pp.

Piggott, M. D., P. E. Farrell, C. R. Wilson, G. J. Gorman, and C. C. Pain, 2009: Anisotropic mesh adaptivity for multi-scale ocean modelling. Philos. Trans. Roy. Soc. A, 367, 4591-4611, doi:10.1098/rsta.2009.0155.

, C. C. Pain, G. J. Gorman, D. P. Marshall, and P. D. Killworth, 2008: Unstructured adaptive meshes for ocean modeling. Ocean Modeling in an Eddying Regime, Geophys. Monogr., Vol. 177, Amer. Geophys. Union, 383-408.

Ringler, T. D., and D. A. Randall, 2002: A potential enstrophy and energy conserving numerical scheme for solution of the shallow-water equations on a geodesic grid. Mon. Wea.
Rev., 130, 1397-1410, doi:10.1175/1520-0493(2002)130<1397: APEAEC $>2.0 . \mathrm{CO} ; 2$.

D. Jacobsen, M. Gunzburger, L. Ju, D. Michael, and W. Skamarock, 2011: Exploring a multiresolution modeling approach within the shallow-water equations. Mon. Wea. Rev., 139, 3348-3368, doi:10.1175/MWR-D-10-05049.1.

, M. Petersen, R. Higdon, D. Jacobsen, P. Jones, and M. Maltrud, 2013: A multiresolution approach to global ocean modeling. Ocean Modell., 69, 211-232, doi:10.1016/ j.ocemod.2013.04.010.

Salmon, R., 1998: Lectures on Geophysical Fluid Dynamics. Oxford University Press, $400 \mathrm{pp}$.

Skamarock, W. C., J. B. Klemp, M. G. Duda, L. D. Fowler, S.-H. Park, and T. D. Ringler, 2012: A multiscale nonhydrostatic atmospheric model using centroidal Voronoi tesselations and C-grid staggering. Mon. Wea. Rev., 140, 3090-3105, doi:10.1175/ MWR-D-11-00215.1.

Taylor, M., J. Tribbia, and M. Iskandarani, 1997: The spectral element method for the shallow water equations on the sphere. J. Comput. Phys., 130, 92-108, doi:10.1006/ jcph.1996.5554.

Trefethen, L. N., 1982: Group velocity in finite difference schemes. SIAM Rev., 24, 113-136.

Ullrich, P. A., and C. Jablonowski, 2011: An analysis of 1D finite-volume methods for geophysical problems on refined grids. J. Comput. Phys., 230, 706-725, doi:10.1016/ j.jcp.2010.10.014.

Vichnevetsky, R., 1987: Wave propagation and reflection in irregular grids for hyperbolic equations. Appl. Numer. Math., 3, 133-166, doi:10.1016/0168-9274(87)90009-2.

Williamson, D. L., J. B. Drake, J. J. Hack, R. Jakob, and P. N. Swarztrauber, 1992: A standard test set for numerical approximations to the shallow water equations in spherical geometry. J. Comput. Phys., 102, 211-224, doi:10.1016/ S0021-9991(05)80016-6. 